Fernanda Garcia Gabira

Gabriela Oliveira ${ }^{1}$

Elizabete Regina Araújo Oliveira ${ }^{1}$

\section{Social determinants of alcohol consumption by adolescents}

\title{
Determinantes sociais do consumo de bebidas alcoólicas por adolescentes
}

\begin{abstract}
Introduction: The consumption of alcoholic beverages in adolescence is a multi-determinate phenomenon, identiffing them either by the individual or social form allows a better understanding of the factors that favor this consumption, such factors can be analyzed together as Social Determinants of Health. Objective: The aim of the present study is to conduct a systematic literature review of articles about health social-determinants associated with alcohol consumption among adolescents. Methods: Articles published between 2008 and 2018 were searched in journals published in Portuguese and English indexed in the Biblioteca Virtual em Saúde (BVS) and Biomedical L iterature from Medical Literature Analysis and Retrieval System Online (PubMed) databases. Results:

In total, 97 articles were selected among systematically edited editions. Variables found in the literature concerning alcohol consumption among adolescents were sex, skin color (race), socioeconomic factors, residence in high social vulnerability areas, living with the father and with other family members without the mother, less concentrated poverty, bigh schooling level, less academic involvement, better housing conditions, having someone to buy alcoholic beverages, having a drinking partner, having parents favorable to alcohol drinking, drinking in family contexts, having alcohol-consumerparents, parental supervision or poor monitoring. Conclusion:

Social determinants significantly associated with alcohol consumption by adolescents were identified. Alcohol consumption in this population shows the urgency of implementing public policies focused on bealth and on understanding the epidemiological scenario of this disease in order to reduce its rates, to provide qualified attention to individuals and, consequenth, to adolescents' bealth.
\end{abstract}

Keywords | Health Social determinants; Adolescents; Social conditions; Socioeconomic factors; Social class.
RESUMO | Introdução: O consumo de bebidas alcoólicas na adolescência é um fenômeno multideterminado, identifica-los seja pela forma individual ou social permite a melhor compreensão dos fatores que favorecem este consumo, tais fatores podem ser analisados conjuntamente como Determinantes Sociais da Saúde. Objetivo: O presente estudo objetivou realizar uma revisão sistemática de artigos publicados sobre os determinantes sociais da saúde associados ao consumo de bebidas alcoólicas por adolescentes. Métodos: Foram pesquisados artigos publicados entre os anos de 2008 e 2018, em periódicos com idiomas português e inglês nas bases de dados da Biblioteca Virtual em Saúde (BVS) e da Biomedical Literature from Medical Literature Analysis and Retrieval System Online (PubMed). Resultados: Foram incluídos na presente revisão sistemática 97 artigos. Os fatores encontrados na literatura que estiveram presentes para o consumo de bebidas alcoólicas entre os adolescentes foram: gênero, raça-cor, socioeconômico, viver em áreas de maior vulnerabilidade social, residir com o pai ou outro familiar que não a mãe, viver com menor concentração de pobreza, alto nível educacional dos pais, baixo envolvimento escolar, menor desempenho acadêmico, melhores condições de moradia, ter irmão consumidor, ter colegas consumidores, ter pais favoráveis, beber em contextos familiares, ter pais consumidores, supervisão parental ou monitoramento deficiente acentuou o consumo. Conclusão: $\mathrm{O}$ presente estudo possibilitou a identificação de determinantes sociais significativamente associados ao consumo de álcool por adolescentes. O consumo de álcool presente nessa população evidencia a urgência na implementação de políticas públicas dentro da saúde, a compreensão do cenário epidemiológico desse agravo a fim de reduzir essa taxa, prestar uma atenção qualificada aos indivíduos e, consequentemente, as consequências para a saúde do adolescente.

Palavras-chave| Determinantes sociais da saúde; Adolescentes; Condições sociais; Fatores socioeconômicos; Classe social.

${ }^{1}$ Universidade Federal do Espírito Santo. Vitória/ES, Brasil. 


\section{INTRODUÇÃO|}

A adolescência compreende uma fase na qual ocorrem intensas transformações e descobertas, resultando em uma progressiva maturidade nos níveis biológicos, físicos, hormonais, cognitivos, sociais, culturais e emocionais que marcam a transição entre a infância e a fase adulta ${ }^{1,2}$. Além das mudanças corporais, o adolescente passa também por alterações comportamentais, tais como crise de identidade, uma suposta rebeldia, isolamento, apego a um grupo, novas formas de se vestir, de se relacionar e de se posicionar ${ }^{2}$, construção de identidade, buscam tornar-se independentes e experimentar novas sensações ${ }^{1}$. Essas transformações comportamentais variam de cultura para cultura e de indivíduo para indivíduo ${ }^{2}$. Essa etapa da vida pode ser considerada uma das mais importantes na transição da vida humana ${ }^{3}$. A combinação de processos biológicos, conduzidos por um estilo de vida moderno e obrigações sociais, podem predispor o adolescente a novas experiências e situações que ameaçam sua saúde ${ }^{1,2}$. Os órgãos governamentais e as publicações divergem quanto ao marco cronológico dessa fase, tanto para seu início como para o seu fim, tendo em vista que essa fase não pressupõe que todos os adolescentes vivem esse período da mesma forma. A Organização Mundial da Saúde (OMS) compreende como adolescente aquele cuja idade está dos 10 aos 19 anos ${ }^{3}$.

Perante todas as drogas, sejam elas lícitas ou ilícitas, a literatura aponta o álcool como a droga mais amplamente utilizada no mundo inteiro ${ }^{4,5}$. No Brasil, esse consumo torna-se hábito, por ter alta aceitabilidade social e por ser frequentemente reforçado ${ }^{6}$. A OMS, em seu relatório sobre álcool e adolescência, demonstrou que esse consumo em indivíduos de idade igual ou superior a 15 anos em nível mundial foi de 6,4 litros de álcool puro por pessoa; já no Brasil esse parâmetro foi de 8,9 litros ${ }^{3}$. O que é mais alarmante ainda é que esse consumo tem início, segundo a literatura, normalmente aos 11-14 anos de idade, e dados demonstram que durante os anos da adolescência essa porcentagem aumenta progressivamente, sobretudo ao final do ensino médio. Tal dado é reforçado pelos estudos realizados no Centro Brasileiro de Informações sobre Drogas Psicotrópicas (CEBRID), que demonstram a tendência mundial à iniciação precoce desse consumo ${ }^{1}$.

Mesmo que a venda seja proibida para menores de 18 anos de idade, assegurado pelo Estatuto da Criança e do Adolescente, no art.81, a venda atinge amplamente os adolescentes, aumentando o índice da experimentação e do consumo alcoólico, preocupando o Ministério da Saúde ${ }^{7}$. Obtêm-se na produção científica ampla variedade dos fatores condicionantes do consumo do álcool pelos adolescentes, nomeadamente fatores de ordem socioeconômica, familiar, meio social, expectativa face o consumo, competências sociais, fatores individuais e de nível psicológico $^{1,8}$, que podem ser analisados conjuntamente como Determinantes Sociais da Saúde (DSS), abordando amplamente a contextualização nesse grupo.

A gênese dos DSS se intensificou em meados da década de 1990, levando posteriormente à criação da Comissão sobre Determinantes Sociais da Saúde (CDSS) em 2004 e da Comissão Nacional de Determinantes Sociais da Saúde (CNDSS) em 2008, abordando a importância do estudo dos DSS para identificar as principais intervenções que devem ser realizadas para alcançar melhores níveis de saúde, educação e desenvolvimento social ${ }^{1}$. Dentre os modelos de análise dos DSS, há a matriz do Institute for Future Studies? utilizado pela CNDSS. Essa matriz está disposta em camadas que evidenciam os possíveis determinantes de saúde e considera tanto os aspectos individuais como os aspectos contextuais e sociais ${ }^{1,10}$.

A predisposição do uso de bebidas alcoólicas está interligada a diferentes fatores ${ }^{1,3}$, e identificá-los seja de forma individual ou social como Determinantes Sociais da Saúde, permite melhor compreensão dos fenômenos que favorecem o consumo de bebidas alcoólicas para estabelecer propostas de intervenção com resolubilidade na prevenção do uso dessa substância. O presente estudo teve como objetivo realizar uma revisão sistemática de artigos publicados sobre os Determinantes Sociais da Saúde associados ao consumo de bebidas alcoólicas por adolescentes.

\section{MÉTODOS|}

Esta revisão sistemática foi orientada pelo seguinte questionamento: "Quais são os determinantes sociais da saúde relativos às condições socioeconômicas, culturais e ambientais gerais, as condições de vida e trabalho, as redes sociais e comunitárias e estilo de vida associados ao consumo de bebidas alcoólicas por adolescentes, presentes na literatura?".

A busca bibliográfica compreendeu-se em duas fontes: Pubmed e Biblioteca Virtual em Saúde (BVS): Medical 
Literature Analysis and Retrieval System Online (MEDLINE), Literatura Latino-Americana e do Caribe em Ciências da Saúde (LILACS), Biblioteca Virtual em Saúde (IBECS) e Scientific Electronic Library Online (SciELO). Os termos presentes no modelo foram localizados no Medical Subject Headings (MeSH) e Descritores em Ciências da Saúde (DeCS) para a confecção das chaves de busca. Para a identificação dos descritores referentes à determinação social do consumo de bebidas alcoólicas a serem utilizados na revisão sistemática, utilizou-se o modelo de determinação social da saúde, proposto por Institute for Future Studies?

A busca foi restrita a artigos publicados entre agosto de 2008 e agosto de 2018, veiculados em periódicos nos idiomas português e inglês. A seleção de artigos não se restringiu aos estudos brasileiros, buscando possibilidades de comparação no uso do referencial teórico utilizado pelos autores, quanto à ampliação do quadro conceitual. Todos os artigos identificados foram exportados de suas fontes para o programa de gerenciamento bibliográfico EndNote Web. As estratégias de busca em cada fonte bibliográfica foram as seguintes:

- BVS: "Adolescente" OR "Adolescent" OR "Adolescentes" OR "Adolescents" OR "Tovem" OR "Teen” OR "Tovens" OR "Teenange" [Descritor de assunto] and "Consumo de bebidas alcoólicas" OR "Alcohol drinking" OR "Alcohol beverages" OR "Bebidas alcoólicas" AND "Condição social" OR "Social conditions" OR "Acesso à informação" OR "Acess to information" OR "Cultura" OR "Culture" OR "Ambiente" OR "Enviroment" OR "Fatores socioeconômicos" OR "Socioeconomic factors" OR "Classe social" OR "Social class" OR "Habitação" OR "Housing" OR "Estilo de vida" OR "Life style" OR "Determinantes sociais da saúde" OR "Social determinants of health" and "HUMANOS, ADOLESCENTES" [Limites].

- Pubmed: "Adolescent" [Mesh Terms] OR "Teen" [Mesh Terms] OR "Teenage" [Mesh Terms] AND ("Alcoholic beverages" [MeSH Terms] OR "Alcohol drinking" [MeSH Terms] AND "Social conditions" [MeSH Terms] OR "Acess to information" [MeSH Terms] OR "Culture" [MeSH Terms] OR "Enviroment" [MeSH Terms] OR "Socioeconomic factors" [MeSH Terms] OR "Social class" [MeSH Terms] OR "Housing" [MeSH Terms] OR "Life style" [MeSH Terms] OR "Social determinants of health" $\mathrm{MeSH}$ Terms]) - Limits Activated: Humans, English, Portuguese, Adolescent, full text, Publication Date from 29/08/2008 to 26/08/2018.

Foram excluídas referências duplicadas, a leitura dos títulos e resumos foi realizada pelos dois primeiros autores do trabalho de maneira independente. Foram considerados elegíveis estudos que tiveram o tipo de exposição relacionada aos determinantes sociais presentes na matriz de Institute for Future Studies ${ }^{9}$, o desfecho de interesse (consumo de bebidas alcoólicas), tipo de estudo e faixa etária da amostra (estudos que incluissem individuos de 10 a 19 anos). Foram critérios de exclusão: estudos referentes à mortalidade por patologias específicas, estudos sem análise estatística adequada para controle de variáveis de confusão e ausência de análise específica, estudos de revisão sistemática, estudos com animais, estudos com universitários e estudos de intervenção. Quando houve divergência entre a dupla de revisores, buscou-se consenso e, quando pertinente, foi realizada a leitura do artigo conjuntamente para decidir sobre a manutenção ou exclusão.

Os artigos selecionados foram lidos em sua íntegra e seus dados, extraídos segundo uma ficha previamente elaborada. Essas informações foram coletadas de cada artigo de maneira independente e comparadas entre a dupla; divergências foram dirimidas através do consenso.

\section{RESULTADOS/DISCUSSÃO|}

Foram identificados 42.224 títulos potenciais para inclusão na revisão sistemática, com 142 duplicadas. A segunda etapa consistiu no total de 358 resumos incluídos, dos resumos lidos, houve concordância de 225 artigos para a inclusão na revisão sistemática desenvolvida de acordo com os critérios do PRISMA Statement ${ }^{11}$ (Preferred Reporting Items for Systematic Reviews and Meta-Analyses) (Figura 1). Após a leitura na íntegra dos artigos, 97 foram incluídos no estudo e 128 excluídos, pois não responderam à pergunta norteadora, estudos qualitativos, artigos de revisão, capítulo de livro, artigos pagos e amostra com outra faixa-etária.

Durante a revisão sistemática foram identificados estudos do tipo transversal (49,4\%), longitudinal (41\%), ensaio-clínicos $(3,1 \%)$, inquéritos populacionais $(4,2 \%)$, e ecológico $(1 \%)$ cujo objetivo foi analisar a associação entre determinantes sociais e o consumo de bebidas alcoólicas por adolescentes.

Do total de artigos analisados, foram identificados 21 fatores associados ao uso de álcool pelos adolescentes. Tais fatores foram agrupados de acordo com os níveis de determinação social da saúde proposto na matriz de Institute for Future Studies?, e vale ressaltar que por ser uma pesquisa que abarca todos os determinantes sociais da saúde que poderiam influenciar no consumo de bebidas presentes na literatura, as pesquisas 
Figura 1 - Fluxograma PRISMA do processo de identificação e seleção dos artigos da revisão sistemática

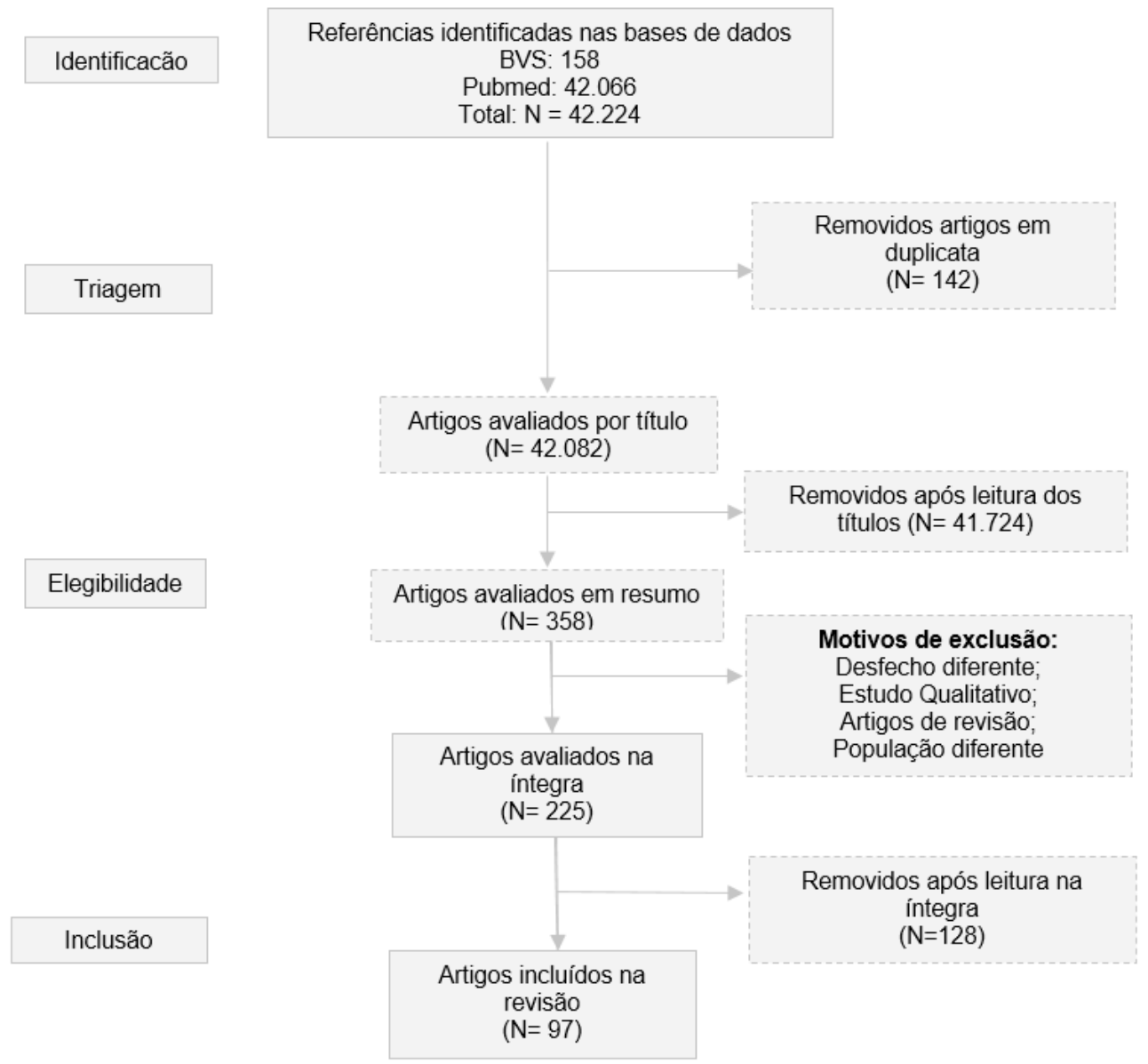

encontradas abordam diferentes fatores de risco com foco em algum fator mais explicitamente, assim a organização para a produção dos resultados se deu no foco da pesquisa em questão mesmo que nela outros determinantes de saúde tenham sido citados.

Vinte e cinco artigos (26,3\%) abordaram o primeiro nível de determinação (condições socioeconômicas, culturais e ambientais gerais) vide Quadro 1. Dezoito artigos (18,9\%) abordam os determinantes referentes às condições de vida e trabalho, vide Quadro 2. E o último nível do modelo, com a junção de estilo de vida, redes comunitárias e sociais, e aspectos sociodemográficos, foi representado pelo maior número de artigos identificados na revisão sistemática, total de 52 artigos (54,7\%), vide Quadro 3.

Como fatores de risco para o consumo de álcool por adolescentes, foram encontrados: adolescentes com renda própria ${ }^{12,30,40}$; alto nível socioeconômico $^{15,16,18,22-27,31,33,3,54,51,84,8,9,91,105}$;baixarenda ${ }^{19,28,29,32,52}$; adolescentes que residem em área com menor vulnerabilidade social $^{28,51}$; raça-cor branca ${ }^{20,22,30,43,72,86,89,90,94,99,104}$; negro ${ }^{94}$; consumo por ambos os pais $12,14,50,5,5,59,61,62,64,646-68,70,79,83,84,102$; apenas a mãe consumidora ${ }^{82}$; apenas o pai consumidor ${ }^{60,82}$; irmãos consumidores ${ }^{55,83,101}$; não ter amigos $^{96}$; amigos/ pares consumidores ${ }^{12,13,20,51,53,55,62,74,77,83,85,86,89,91,92,98,100,102,103}$; por sexo evidencia-se a prevalência para meninos ${ }^{12,15,20,21,25,28,30,33,34,37,39,51,52,57,59,65,68,69,71,89,90,97,105}$; meninas 61,79,90,92,95; pais com alta escolaridade ${ }^{15,39,34,75}$; mães com alta escolaridade ${ }^{38}$; baixa escolaridade dos pais ${ }^{46}$; baixo apoio familiar ${ }^{16}$; monitoramento prejudicado ${ }^{55,56,80,89,100}$; divórcio ${ }^{61,62}$; relacionamento conflituoso com os pais ${ }^{86,91}$; residir apenas com o pai ${ }^{15,95}$; pais menos exigentes ${ }^{32}$; baixo desempenho escolar ${ }^{68,89}$; sem vínculo escolar ${ }^{100}$; estudar em escola particular ${ }^{16}$ e escola pública ${ }^{29}$; ser ausente nas aulas ${ }^{52}$; não frequentar as aulas ${ }^{30}$; zona escolar urbana ${ }^{17,38,46}$; zona rural $^{23,45}$; estrato geográfico: região sul ${ }^{30}$; viver próximo a ponto de venda $a^{36}$. 
Quadro 1 - Distribuição de artigos segundo determinantes sociais relativos a condições socioeconômicas, culturais e ambientais gerais

\begin{tabular}{|c|c|c|c|c|}
\hline $\begin{array}{l}\text { Autor } \\
\text { (Ano) }\end{array}$ & Local & $\begin{array}{l}\text { Tipo de } \\
\text { estudo }\end{array}$ & $\begin{array}{l}\text { Amostra } \\
\text { (Faixa } \\
\text { etária) }\end{array}$ & Resultado \\
\hline $\begin{array}{l}\text { Chen et al. } \\
(2010)^{12}\end{array}$ & EUA & $\begin{array}{l}\text { Inquérito } \\
\text { domiciliar }\end{array}$ & $\begin{array}{c}109 \\
(14 \text { a } 16)\end{array}$ & $\begin{array}{l}\text { Relacionadas positivamente com a frequência de consumo por: } \\
\text { idade, consumo percebido pelos pais, pelos pares, renda pessoal } \\
\text { e a amizade, maior entre os meninos; Adolescentes com carro e } \\
\text { amigos que tinham carro não tiveram associação }(p<0,001) \text {. }\end{array}$ \\
\hline $\begin{array}{l}\text { Huckle et al. } \\
(2008)^{13}\end{array}$ & EUA & $\begin{array}{l}\text { Inquérito } \\
\text { telefônico }\end{array}$ & $\begin{array}{c}1179 \\
(12 \text { a } 17)\end{array}$ & $\begin{array}{c}\text { Não encontrou associações com relação as condições } \\
\text { socioeconômicas. Os amigos foram identificados como o grupo } \\
\text { que fornecia álcoo1l aos jovens com mais frequência }\end{array}$ \\
\hline $\begin{array}{l}\text { Jorge et al. } \\
(2017)^{14}\end{array}$ & BRASIL & Longitudinal & $\begin{array}{l}117.547 \text { e } \\
25.569 \\
(15 \text { a } 19)\end{array}$ & $\begin{array}{l}\text { Maior chance de aumento do consumo excessivo de álcool entre } \\
\text { os adolescentes que viviam em áreas de maior vulnerabilidade } \\
\text { social, mães }(p<0,001) \text { e pais }(p=0,001) \text { consumidores e fumantes; } \\
\text { modificação no padrão de consumo associaram-se a: religião } \\
\quad(p=0,004) \text {, membro familiar consumidor }(p<0,001) \text {. }\end{array}$ \\
\hline $\begin{array}{l}\text { Lu et al. } \\
(2015)^{15}\end{array}$ & CHINA & Transversal & 13.811 & $\begin{array}{l}\text { P valor maior no sexo masculino, chance aumentada para o } \\
\text { nível de escolaridade, e com o pai ou outro familiar que não a } \\
\text { mãe. E alunos com socioeconômico mais alto possuíram maior } \\
\text { probabilidade de consumo comparado aos do nível baixo. }\end{array}$ \\
\hline $\begin{array}{l}\text { Andrade } \\
(2013)^{16}\end{array}$ & EUA & Longitudinal & $\begin{array}{c}6.228 \\
(7 \text { a } 22)\end{array}$ & $\begin{array}{l}\text { Prevalência aos } 12 \text { anos de idade; estudantes pertencentes a } \\
\text { famílias com baixo apoio familiar/parental; Alunos que frequentam } \\
\text { escolas particulares, escolas maiores e escolas com menores } \\
\text { concentrações de pobreza (no caso de escolas públicas) eram } \\
\text { mais propensos a ter um nível maior de uso de álcool na linha } \\
\text { de base. O efeito do apoio dos pais é mais protetor no caso de } \\
\text { estudantes que frequentam escolas com níveis mais altos de } \\
\text { pobreza. }\end{array}$ \\
\hline $\begin{array}{l}\text { Azar et al. } \\
(2016)^{17}\end{array}$ & AUSTRÁLIA & Longitudinal & $\begin{array}{c}68.208 \\
(12 \text { a } 17)\end{array}$ & $\begin{array}{c}\text { Densidade de saída de álcool é maior }(2 x) \text { em áreas regionais em } \\
\text { comparação as áreas urbanas. }\end{array}$ \\
\hline $\begin{array}{l}\text { Caria et al. } \\
(2011)^{18}\end{array}$ & EUROPA & $\begin{array}{l}\text { Ensio clínico } \\
\text { randomizado } \\
\text { controlado }\end{array}$ & $\begin{array}{c}7.079 \\
(12 \text { a } 14)\end{array}$ & $\begin{array}{l}\text { Alunos de escolas de alto nível socioeconômico foram mais } \\
\text { propensos a consumir álcool do que estudantes de outras escolas } \\
\text { de beber pelo menos uma vez por mês }(17,2 \% \text { vs. } 14,6 \%, p= \\
\text { 0,01) e ter intenção de beber }(43,7 \% \text { vs. } 39,0 \%, p<0,01) \text {. }\end{array}$ \\
\hline $\begin{array}{l}\text { Choukas- } \\
\text { Bradley } \\
\text { et al. }(2015)^{19}\end{array}$ & EUA & Transversal & $\begin{array}{c}364 \\
(14 \text { a } 18)\end{array}$ & $\begin{array}{l}\text { Aumento do uso de álcool nas séries no nono e décima segunda } \\
\text { série, ou seja, níveis mais altos de preferência social; adolescentes } \\
\text { pertencentes a família com socioeconômico mais baixo e com } \\
\text { níveis mais altos de violência; masculino + popularidade e } \\
\text { caucasianos. }\end{array}$ \\
\hline $\begin{array}{l}\text { Brenner } \\
\text { et al. }(2011)^{20}\end{array}$ & EUA & Longitudinal & $\begin{array}{c}839 \\
(16,5 \pm 0,66 \\
\text { e } 17,8 \pm \\
0,64)\end{array}$ & $\begin{array}{l}\text { Individual: Brancos bebiam mais que negros; sexo masculino } \\
\text { bebe mais que feminino; uso pelos pares + ensino médio; idade, } \\
\text { socioeconômico e escolaridade materna não foram significativas; } \\
\text { Maior apoio materno reduziu o consumo de álcool; uso do álcool } \\
\text { não foi associado a desvantagem econômica da região. }\end{array}$ \\
\hline $\begin{array}{l}\text { Chen et al. } \\
(2012)^{21}\end{array}$ & EUA & Transversal & $\begin{array}{c}46.675 \\
(12 \text { a } 17)\end{array}$ & $\begin{array}{l}\text { Prevalência ao sexo masculino; idade início aos } 14 \text { anos; pessoas } \\
\text { com nove ou mais anos de estudo, apresentou maior prevalência } \\
\text { de consumo comparado aos com oito anos de estudo; maior } \\
\text { escolaridade teve maior prevalência comparado aos com menor } \\
\text { escolaridade para o consumo alcoólico. }\end{array}$ \\
\hline
\end{tabular}


*continuação.

\begin{tabular}{|c|c|c|c|c|}
\hline $\begin{array}{l}\text { Clark et al. } \\
(2013)^{22}\end{array}$ & EUA & Longitudinal & $\begin{array}{c}15.278 \\
(11 \text { a } 21)\end{array}$ & $\begin{array}{l}\text { Menos propensos a beber foram os negros; a quantidade de uso } \\
\text { de álcool aumenta entre os brancos em níveis de renda mais altos. } \\
\text { Da mesma forma, entre os brancos, a probabilidade de consumo } \\
\text { excessivo aumentou à medida que o nível de renda domiciliar } \\
\text { aumentou. Os negros seguem um padrão similar de aumento de } \\
\text { consumo com maior renda. Da mesma forma, a probabilidade de } \\
\text { um participante negro beber todos os dias aumentou de } 0,006 \text { para } \\
0,012 \text { quando a renda subiu do nível mais baixo ( }<16.000 \text { dólares) } \\
\text { para o nível mais alto de renda }(>\$ 60.000) \text {. Adolescentes que } \\
\text { viviam com ambos os pais eram menos propensos a beber. }\end{array}$ \\
\hline $\begin{array}{l}\text { Coomber } \\
\text { et al. }(2011)^{23}\end{array}$ & $\begin{array}{l}\text { AUSTRÁLIA E } \\
\text { EUA }\end{array}$ & Transversal & $\begin{array}{c}3.729 \\
(12 \text { a } 15)\end{array}$ & $\begin{array}{l}\text { Taxas de consumo de álcool atual foram significativamente mais } \\
\text { altas nas áreas rurais em comparação aos estudantes urbanos } \\
(\mathrm{OR}=1,31) \text {. Maior socioeconômico familiar teve efeito protetor na } \\
\text { redução do comportamento de uso de substâncias com } \mathrm{OR}=0,40 \text {. }\end{array}$ \\
\hline $\begin{array}{l}\text { Hill e Mrug } \\
(2015)^{24}\end{array}$ & EUA & Transversal & $\begin{array}{c}23.615 \\
(12 \text { a } 18)\end{array}$ & $\begin{array}{c}\text { A pobreza no nível da escola, mais estudantes de minorias étnicas, } \\
\text { baixo aproveitamento e maior absenteísmo foram relacionados ao } \\
\text { uso de álcool particularmente em níveis mais baixos. }\end{array}$ \\
\hline $\begin{array}{l}\text { Locatelli } \\
\text { et al. } \\
(2012)^{25}\end{array}$ & BRASIL & Transversal & $\begin{array}{c}2.613 \\
(16 \pm 0,98)\end{array}$ & $\begin{array}{l}\text { O consumo esteve maior entre o sexo masculino e classes sociais } \\
\text { mais altas. O modelo de regressão exibiu uma taxa crescente } \\
\text { de consumo excessivo de álcool com o aumento do status } \\
\text { socioeconômico. }\end{array}$ \\
\hline $\begin{array}{l}\text { Lu et al. } \\
(2016)^{26}\end{array}$ & CHINA & Transversal & $\begin{array}{c}7.075 \\
(15.7 \pm 1.73)\end{array}$ & $\begin{array}{l}\text { Achados demonstram que estudantes de nível socioeconômico } \\
\text { local mais elevado tem uma prevalência crescente no } \\
\text { comportamento de beber. }\end{array}$ \\
\hline $\begin{array}{l}\text { Lui et al. } \\
(2015)^{27}\end{array}$ & EUA & Longitudinal & $\begin{array}{c}7.123 \\
(12 \text { a } 32)\end{array}$ & $\begin{array}{c}\text { Entre brancos e negros, grupos econômicos e de capital humano } \\
\text { menores relataram menor uso-não uso. }\end{array}$ \\
\hline $\begin{array}{l}\text { Martins- } \\
\text { Oliveira } \\
\text { et al. }(2016)^{28}\end{array}$ & BRASIL & Transversal & $\begin{array}{c}936 \\
(15 \text { a } 19)\end{array}$ & $\begin{array}{l}\text { Adolescentes vulneráveis socioeconômicamente apresentam } \\
\text { maior prevalência de risco ( } p=0,004 \text { ). Sexo masculino mais } \\
\text { propenso ao risco do que sexo feminino (RR ajustado: } 0,75 \text {; IC } \\
95 \% \text { : } 0,54 \text { a 1,0). Participantes de áreas de baixa vulnerabilidade } \\
\text { social (aqueles com maior nível socioeconômico) eram mais } \\
\text { propensos a apresentar uso perigoso para possível dependência } \\
\text { em comparação àqueles que viviam em áreas menos favorecidas } \\
\text { (RR ajustado: } 2,80 ; \text { IC95\%: } 1,14 \text { a } 2,15 \text { ) }\end{array}$ \\
\hline $\begin{array}{l}\text { Mrug et al. } \\
(2010)^{29}\end{array}$ & ALABAMA & Transversal & $\begin{array}{c}542 \\
(13,2 \pm 0,9)\end{array}$ & $\begin{array}{l}\text { Escolas que tinham níveis mais altos de pobreza e mais minorias } \\
\text { raciais/etnicas tinham maiores proporções de estudantes que } \\
\text { endossavam o uso de álcool ( } R=0.44-0.51 \text { ). Entre os fatores } \\
\text { de risco proximais, a variabilidade entre escolas foi maior para o } \\
\text { comportamento desviante dos amigos do que para a parentalidade. }\end{array}$ \\
\hline $\begin{array}{l}\text { Pinsky et al. } \\
(2010)^{30}\end{array}$ & BRASIL & Transversal & $\begin{array}{c}661 \\
(14 \text { e } 17)\end{array}$ & $\begin{array}{l}\text { Idade de ínicio de consumo aos } 14 \text { anos; viver na parte sul do } \\
\text { Brasil, não frequentar a escola e aqueles que estão trabalhando, } \\
\text { e os indivíduos negros e aqueles com renda pessoal relataram } \\
\text { uma maior frequência de consumo de álcool. Fatores } \\
\text { sociodemográficos, como gênero, faixa de renda, renda familiar e } \\
\text { status de estudante aumentam a quantidade de álcool consumida. } \\
\text { Os homens relatam uma maior frequência de consumo excessivo } \\
\text { de álcool do que as mulheres. }\end{array}$ \\
\hline $\begin{array}{l}\text { Poonawalla } \\
\text { et al. }(2014)^{31}\end{array}$ & EUA & Longitudinal & $\begin{array}{l}\text { Fase } 1= \\
1.364 \\
\text { Fase } 2= \\
1.226 \\
\text { (Nascimento } \\
\text { aos } 15 \text { anos }\end{array}$ & $\begin{array}{l}\text { A trajetória de renda familiar foi significativamente associada ao } \\
\text { uso de álcool para sempre }(P=0,020) \text { e ao álcool no último ano } \\
\text { aos } 15 \text { anos }(P=0,008) \text {. }\end{array}$ \\
\hline
\end{tabular}


*continuação.

\begin{tabular}{|c|c|c|c|c|}
\hline $\begin{array}{l}\text { Primack } \\
\text { et al. }(2009)^{32}\end{array}$ & PITTSBURGH & Transversal & $\begin{array}{c}1.402 \\
(14 \text { a } 18)\end{array}$ & $\begin{array}{c}\text { O uso de álcool foi associado com idade mais avançada }(P<0,001) \text {, } \\
\text { menor nível socioeconômico }(P=0,035) \text {, parentalidade menos } \\
\text { exigente }(P<0,001) \text {, parentesco menos responsivo }(P<0,001) \text {, } \\
\text { aumento do estresse }(P=0.001) \text {, aumento da procura de } \\
\text { sensações }(P<0,001) \text {, aumento da rebeldia }(P<0,001) \text {, aumento } \\
\text { da depressão }(P<0,001) \text {, menor autoestima }(P=0,045) \text { e menor } \\
\text { aproveitamento escolar }(P<001) .\end{array}$ \\
\hline $\begin{array}{l}\text { Fagan } \\
(2015)^{33}\end{array}$ & CHICAGO & Longitudinal & $\begin{array}{c}1.416 \\
(9,12 \text { e } 15)\end{array}$ & $\begin{array}{l}\text { Sexo masculino foi mais propenso ao consumo em todas } \\
\text { as ondas, caucasianos beberam mais que afro-americanos. } \\
\text { Adolescentes com cuidadores de com salários mais altos tiveram } \\
\text { maior probabilidade de consumo excessivo comparado aos de } \\
\text { baixa renda. O coeficiente positivo indica que a relação entre } \\
\text { vitimização e uso de álcool era mais forte para aqueles que } \\
\text { moravam em bairros com níveis de desvantagem mais elevados } \\
\text { versus menores. }\end{array}$ \\
\hline $\begin{array}{l}\text { Ferreira } \\
\text { et al. }(2011)^{34}\end{array}$ & BRASIL & Transversal & $\begin{array}{l}284 \\
(14)\end{array}$ & $\begin{array}{l}\text { Prevalência no sexo masculino; pessoas com nove ou mais anos } \\
\text { de estudo há maior prevalência de consumo comparado com os } \\
\text { de até oito anos de estudo, também maior quantidade de consumo } \\
2 x \text { ao mês para os com maior renda comparados aos com baixa } \\
\text { renda. }\end{array}$ \\
\hline $\begin{array}{l}\text { Sweeting } \\
\text { e Hunt } \\
(2015)^{35}\end{array}$ & ESCOCIA & Transversal & $\begin{array}{c}2.937 \\
(13 \text { a } 15)\end{array}$ & $\begin{array}{l}\text { Beber esteve associado à privação residencial; afluência familiar, } \\
\text { e socioeconômico não houve associação ao consumo de álcool. }\end{array}$ \\
\hline $\begin{array}{l}\text { Young et al. } \\
(2013)^{36}\end{array}$ & ESCÓCIA & Transversal & $\begin{array}{l}979 \\
(15)\end{array}$ & $\begin{array}{l}\text { Alunos que vivem próximos (a menos de } 200 \mathrm{~m} \text { ) de um ponto de } \\
\text { venda fora do mercado tinham quase o dobro de probabilidade } \\
\text { de beber semanalmente do que os que moravam a mais de } \\
800 \mathrm{~m} \text { de tais pontos de venda ( } p \leq 004 \text { ). Alunos que vivem em } \\
\text { áreas com alta densidade de estabelecimentos off-sales nas } \\
\text { proximidades ( } 31+\text { em } 1200 \mathrm{~m} \text { ) tinham aproximadamente } 50 \% \\
\text { mais probabilidades de beber semanalmente do que aqueles } \\
\text { com poucos }(0-10) \text { pontos de venda dentro de } 1200 \mathrm{~m}(\mathrm{P} \leq 05) \text {. } \\
\text { Não houve interações significativas entre gênero, proximidade de } \\
\text { saída ou densidade. }\end{array}$ \\
\hline
\end{tabular}

Quadro 2 - Distribuição de artigos segundo condições de vida, trabalho, educação, emprego e habitação

\begin{tabular}{|c|c|c|c|c|}
\hline $\begin{array}{l}\text { Autor } \\
\text { (Ano) }\end{array}$ & Local & $\begin{array}{l}\text { Tipo de } \\
\text { estudo }\end{array}$ & $\begin{array}{c}\text { Amostra } \\
\text { Faixa etária }\end{array}$ & Resultado \\
\hline $\begin{array}{l}\text { Brody et al. } \\
(2012)^{37}\end{array}$ & EUA & Longitudinal & $\begin{array}{l}538 \\
(11)\end{array}$ & $\begin{array}{c}\text { Aumento do consumo do álcool com o decorrer da idade (16 a } \\
18 \text { anos); sexo masculino ( } p<0,005) \text {; interação para gênero e } \\
\text { discriminação na predição do uso de bebida }(p<0,001) \text { influência } \\
\text { significativa para o sexo masculino }(O R=1,11 ; p<0,001) \text {. Também } \\
\text { para envolvimento escolar }(p<0,05) .\end{array}$ \\
\hline $\begin{array}{l}\text { Brito et al. } \\
(2015)^{38}\end{array}$ & BRASIL & Transversal & $\begin{array}{c}4.269 \\
(14 \text { e } 19)\end{array}$ & $\begin{array}{l}\text { Escolaridade materna, residentes de área urbana e regiões semi- } \\
\text { árida tiveram chance de exposição de três ou mais risco ao uso } \\
\text { do álcool; mãe com escolaridade de } 9 \text { a } 11 \text { anos tiveram filhos } \\
\text { com mais chance de risco comparados as mães com menor } \\
\text { escolaridade. }\end{array}$ \\
\hline $\begin{array}{l}\text { Berten et al. } \\
(2012)^{39}\end{array}$ & BRUXELAS & Transversal & $\begin{array}{c}2.513 \\
(12 \text { a } 20)\end{array}$ & $\begin{array}{l}\text { Meninos e estudantes mais velhos e estudantes nativos usam } \\
\text { álcool; Os usuários frequentes de álcool são mais frequentemente } \\
\text { encontrados em crianças de pais com nível educacional alto. } \\
\text { Independentemente da origem étnica/cultural de um aluno, o } \\
\text { crescimento em uma "família de alta escolaridade" aumentou o } \\
\text { risco de uso frequente de álcool. }\end{array}$ \\
\hline
\end{tabular}


*continuação.

\begin{tabular}{|c|c|c|c|c|}
\hline $\begin{array}{l}\text { Bosque- } \\
\text { Prous et al. } \\
(2017)^{40}\end{array}$ & EUROPA & Transversal & $\begin{array}{c}8.705 \\
(14 \text { e } 17)\end{array}$ & $\begin{array}{l}\text { No entanto, o consumo compulsivo semanal foi menos prevalente } \\
\text { em adolescentes com alto desempenho acadêmico do que } \\
\text { aqueles com baixo desempenho (RP }=0,34 ; \text { IC95\% }=0,14 \square 0,87 \text { ) } \\
\text { e mais prevalente em adolescentes com renda semanal de } € 50 \\
\text { comparado àqueles com } \leq € 5 / \text { semana ( } R P=3,14 ; \text { IC95\% }=2,23- \\
4,42) \text {. Essas associações variavam de acordo com o país, mas } \\
\text { não de acordo com o gênero ou faixa etária. }\end{array}$ \\
\hline $\begin{array}{l}\text { Andreas e } \\
\text { Jackson } \\
(2015)^{41}\end{array}$ & EUA & Longitudinal & $\begin{array}{l}891 \\
(12)\end{array}$ & $\begin{array}{l}\text { O ensino médio aumentou a chance de uso. Além disso, a } \\
\text { delinquência precoce surgiu como um fator de risco tal que as } \\
\text { diferenças no uso de álcool existiam antes da transição. }\end{array}$ \\
\hline $\begin{array}{l}\text { Chung et al. } \\
(2014)^{42}\end{array}$ & PITTSBURGH & Longitudinal & $\begin{array}{c}2.451 \\
(11 \text { a } 18)\end{array}$ & $\begin{array}{l}\text { A prevalência foi maior entre as meninas brancas, em relação às } \\
\text { negras, com idades entre } 11 \text { e } 18 \text { anos; Entre os preditores, a } \\
\text { facilidade de acesso percebida ao álcool }(p<0,001) \text {, vizinhança de } \\
\text { risco (ou seja, testemunhar a venda de drogas) }(p<0,001) \text {. }\end{array}$ \\
\hline $\begin{array}{l}\text { D'Amico } \\
\text { et al. } \\
(2016)^{43}\end{array}$ & CALIFÓRNIA & Longitudinal & $\begin{array}{c}6.509 \\
(11 \text { a } 17)\end{array}$ & $\begin{array}{l}\text { Jovens brancos relatando uma maior interceptação geral do uso } \\
\text { de álcool em comparação com todos os outros grupos }(p<0,001 \text {, } \\
p=0,001, p=0,008) \text {. Interceptação de álcool relataram maior } \\
\text { despreparo acadêmico }(p<0,001) \text { e inadimplência }(p<0,001) \text {. }\end{array}$ \\
\hline $\begin{array}{l}\text { Davies et al. } \\
(2017)^{44}\end{array}$ & HOLANDA & Transversal & $\begin{array}{c}191 \\
(15 \text { a } 20)\end{array}$ & $\begin{array}{l}\text { Baixo nível educacional tinha maior probabilidade de beber muito } \\
(\mathrm{OR}=3,25, \quad \text { IC } 95 \%=1,48-7,17) \text { do que adolescentes na faixa } \\
\text { educacional alta. }\end{array}$ \\
\hline $\begin{array}{l}\text { Donath } \\
\text { et al. } \\
(2011)^{45}\end{array}$ & ALEMANHA & Transversal & $\begin{array}{c}44.610 \\
(15,3 \pm 0,7)\end{array}$ & $\begin{array}{l}\text { A prevalência em tempo de vida para o consumo de álcool difere } \\
\text { significativamente entre áreas rurais }(93,7 \%) \text { e áreas urbanas } \\
(86,6 \%) \text { das grandes cidades; }(89,1 \%) \text { cidades menores com } \\
\text { maior prevalência nas áreas rurais. }\end{array}$ \\
\hline $\begin{array}{l}\text { Ennett et al. } \\
(2008)^{46}\end{array}$ & EUA & Longitudinal & $\begin{array}{c}6.544 \\
(11 \text { a } 17)\end{array}$ & $\begin{array}{l}\text { Vizinhança }(p<0,001) \text { em torno do intercepto médio para uso } \\
\text { de álcool centrado aos } 12 \text { anos. A matrícula no ensino médio, } \\
\text { associou-se significativamente positivamente às trajetórias de } \\
\text { abuso de álcool }(p<0,0001) \text {. Níveis iniciais de abuso de álcool } \\
\text { foram maiores apenas para jovens cujo pais tinha inferior em vez } \\
\text { de níveis mais elevados de ensino }(p<.05) \text {. A taxa de mudança } \\
\text { no abuso de álcool foi mais lenta para a juventude negra em } \\
\text { comparação com a juventude branca (B }=-0,6 \text {, SE }=0,01 \text {, } \\
p<0,0001) \text { e mais rápido para os jovens em famílias sem dois pais } \\
(B=0,04, S E=0,01, p<0,01) .\end{array}$ \\
\hline $\begin{array}{l}\text { Xianfang } \\
\text { et al. } \\
(2014)^{47}\end{array}$ & MICHIGAN & Transversal & $\begin{array}{c}12.341 \\
(17 \text { e } 18)\end{array}$ & $\begin{array}{l}\text { Associações entre estresse no trabalho, aspiração acadêmica, } \\
\text { influência dos pais e influência dos pares com o uso de álcool; }\end{array}$ \\
\hline $\begin{array}{l}\text { Malta et al. } \\
(2014)^{48}\end{array}$ & BRASIL & Transversal & $\begin{array}{c}109.104 \\
(13 \text { a } 15)\end{array}$ & $\begin{array}{l}\text { Não houve diferenças entre estudantes de escolas públicas e } \\
\text { privadas. Relatam problemas com amigos ou família devido o } \\
\text { consumo de álcool. Consumiam principalmente em festas com } \\
\text { amigos, aquisição através de mercado, lojas ou bares; casa. }\end{array}$ \\
\hline $\begin{array}{l}\text { Mejia et al. } \\
(2016)^{49}\end{array}$ & $\begin{array}{l}\text { ARGENTINA } \\
\text { E MÉXICO }\end{array}$ & Transversal & $\begin{array}{c}13.295 \\
(12,5 \pm 0,7)\end{array}$ & $\begin{array}{l}\text { O consumo de álcool também foi maior entre os estudantes } \\
\text { argentinos do que os mexicanos }(p=0,003) \text { em ambos os países. }\end{array}$ \\
\hline $\begin{array}{l}\text { Noal } \\
(2010)^{50}\end{array}$ & BRASIL & Longitudinal & $\begin{array}{c}4.452 \\
(11,3 \pm 0,3)\end{array}$ & $\begin{array}{l}\text { Houve associação direta entre o uso experimental de álcool e o } \\
\text { consumo parental de álcool }(p<0,001) \text {. Repetição escolar não foi } \\
\text { estatisticamente associada ao desfecho do estudo. }\end{array}$ \\
\hline
\end{tabular}


*continuação.

\begin{tabular}{|c|c|c|c|c|}
\hline $\begin{array}{l}\text { Zarzar et al. } \\
(2012)^{51}\end{array}$ & BRASIL & Transversal & $\begin{array}{c}891 \\
(15-19)\end{array}$ & $\begin{array}{l}\text { O consumo compulsivo de álcool foi associado ao sexo masculino } \\
\text { (OR=1,52; IC95\% } 1,01-2,28) \text { e a viver em uma área de baixa } \\
\text { vulnerabilidade (melhores condições de moradia, escolaridade, } \\
\text { renda, trabalho, assistência jurídica e saúde) (OR=1,66). Os } \\
\text { estudantes que relataram que seus amigos mais próximos } \\
\text { eram da escola (em oposição aos amigos da igreja) tinham um } \\
\text { risco aumentado de consumo excessivo de álcool (OR=3,55, IC } \\
99 \% 1,91-5,87) .\end{array}$ \\
\hline $\begin{array}{l}\text { Zenic et al. } \\
(2015)^{52}\end{array}$ & $\begin{array}{l}\text { BÓSNIAE } \\
\text { HERZEGOV- } \\
\text { INA }\end{array}$ & Transversal & $\begin{array}{c}970 \\
17 \text { a } 18\end{array}$ & $\begin{array}{l}\text { Gênero masculino, mais ausentes da escola }(\mathrm{OR}=1,664 ; \mathrm{IC} 95 \% \\
=1.328-2.085) \text {, com menor nível financeiro da família }(\mathrm{OR}= \\
0,495 ; \mathrm{IC} 95 \%=0,255-0,963) \text { e maior escolaridade da mãe }(\mathrm{OR}= \\
1,403 ; \mathrm{IC} 95 \%=1,079-1,823) .\end{array}$ \\
\hline $\begin{array}{l}\text { Botticello } \\
(2013)^{53}\end{array}$ & EUA & Longitudinal & $\begin{array}{l}10.574 \\
(11 \text { a } 17)\end{array}$ & $\begin{array}{c}\text { Uso pelos pares com associação a maior probabilidade de uso } \\
\text { ao longo da vida; vínculo escolar está associado a uma menor } \\
\text { probabilidade de uso ao longo da vida. }\end{array}$ \\
\hline $\begin{array}{l}\text { Lo et al. } \\
(2013)^{54}\end{array}$ & ALABAMA & Transversal & $\begin{array}{c}92.822 \\
(14,5 \pm 2,0)\end{array}$ & $\begin{array}{l}\text { Demonstrou que a escola que desempenha um forte papel } \\
\text { protetor reduziu significativamente não apenas o uso recente de } \\
\text { substâncias por seus próprios alunos, mas também de estudantes } \\
\text { nas SCAs vizinhas. }\end{array}$ \\
\hline
\end{tabular}

Quadro 3 - Distribuição de artigos segundo estilo de vida, redes comunitárias, sociais, idade e sexo

\begin{tabular}{|c|c|c|c|c|}
\hline $\begin{array}{l}\text { Autor } \\
\text { (Ano) }\end{array}$ & Local & $\begin{array}{l}\text { Tipo de } \\
\text { estudo }\end{array}$ & $\begin{array}{c}\text { Amostra } \\
\text { Faixa etária }\end{array}$ & Resultados \\
\hline $\begin{array}{l}\text { Chan et al. } \\
(2013)^{55}\end{array}$ & AUSTRÁLIA & Transversal & $\begin{array}{c}808 \\
(11 \text { a 17) }\end{array}$ & $\begin{array}{l}\text { Quando comparado aos não usuários, ter irmão e colegas que } \\
\text { consumem álcool levou ao aumento acentuado em todos os níveis } \\
\text { escolares; pais favoráveis a beber foram associadas ao consumo } \\
\text { acentuado em relação aos não-bebedores, assim como a atitude } \\
\text { dos pais; e supervisão parental deficiente. Quando começa em } \\
\text { idade precoce uso pelos pares torna-se predominante. }\end{array}$ \\
\hline $\begin{array}{l}\text { Fulkerson } \\
\text { et al. } \\
(2008)^{56}\end{array}$ & EUA & Transversal & $\begin{array}{c}4259 \\
(11,8 \pm 0,57)\end{array}$ & $\begin{array}{l}\text { Pais sobre o controle social informal relacionado ao álcool } \\
\text { foram significativa e positivamente relacionadas aos relatos de } \\
\text { monitoramento dos pais, e que o monitoramento das atividades } \\
\text { das crianças pelos pais foi significativamente inversamente } \\
\text { relacionado ao usoe intenções deálcool. controles sociais informais } \\
\text { para reduzir o consumo de álcool em bairros estão relacionadas } \\
\text { a níveis mais altos de monitoramento parental e, por sua vez, a } \\
\text { níveis mais baixos de uso e intenções adolescentes de álcool; } \\
\text { Renda mais baixa, área urbana, sugerem que as percepções dos } \\
\text { pais sobre o controle social e seu próprio monitoramento estão } \\
\text { significativamente associadas. }\end{array}$ \\
\hline $\begin{array}{l}\text { Ghandour } \\
\text { et al. } \\
(2015)^{57}\end{array}$ & LÍBANO & Transversal & $\begin{array}{l}5.109 \text { e } \\
2.784 \\
(13,80 \pm \\
0,02 \text { e } 13,91 \\
\pm 0,02)\end{array}$ & $\begin{array}{l}\text { Adolescentes do sexo masculino eram mais propensos a obter } \\
\text { álcool de "lojas" ou "através de seus amigos", enquanto a principal } \\
\text { fonte de mulheres era sua "família". Um em cada vinte relatou } \\
\text { problemas relacionados ao álcool (por exemplo, entrar em brigas } \\
\text { com a família / amigos e pular escola). }\end{array}$ \\
\hline $\begin{array}{l}\text { Gruenewald } \\
\text { et al. } \\
(2015)^{58}\end{array}$ & $\begin{array}{c}\text { NOVA } \\
\text { ZELÂNDIA }\end{array}$ & Transversal & $\begin{array}{c}13.243 \\
(14 \text { a } 19)\end{array}$ & $\begin{array}{l}\text { A idade foi associada com o consumo mais frequente de pubs/ } \\
\text { casas noturnas entre os jovens de } 18-19 \text { anos }(P=0,009) \text { e } \\
\text { maiores quantidades consumidas em casa }(P=0,010) \text { e casas dos } \\
\text { outros }(P=0,029) \text {. A frequência e a quantidade de bebida na faixa } \\
\text { etária de } 16 \text { a } 17 \text { anos aumentaram em casa }(P=0,040, P=0,002) \\
\text { e outras casas }(P=0,002, P=0,021) .\end{array}$ \\
\hline
\end{tabular}


*continuação.

\begin{tabular}{|c|c|c|c|c|}
\hline $\begin{array}{l}\text { Haugland } \\
\text { et al. } \\
(2013)^{59}\end{array}$ & NORUEGA & Longitudinal & $\begin{array}{c}50.807 \\
\text { (13 ou mais) }\end{array}$ & $\begin{array}{l}\text { Mais comum em meninos }(O R=1,6 ; I C=1,1-2,3) \text { e meninas }(O R \\
=1,5 ; I C=1,1-2,1) \text { com pais abusadores de álcool versus aqueles } \\
\text { com filhos pais que não abusam; Altos níveis de consumo de álcool } \\
\text { foram menos comuns entre os meninos com mães que abusam do } \\
\text { álcool }(O R=0,2 ; I C=0,1-0,6) \text { em comparação com outros meninos. }\end{array}$ \\
\hline $\begin{array}{l}\text { Hung et. al } \\
(2015)^{60}\end{array}$ & TAIWAN & Longitudinal & $\begin{array}{l}\text { Coorte } 1= \\
2.719 \\
\text { Coorte } 2= \\
2.499 \\
\text { Final }= \\
1.961 \\
(10 \text { a } 11 \\
14 \text { a } 15)\end{array}$ & $\begin{array}{l}\text { O comportamento e a atitude de beber do pai influenciaram } \\
\text { o comportamento de beber do adolescente, ao passo que o } \\
\text { comportamento e a atitude materna de beber não o fizeram. } \\
\text { Adolescentes do sexo masculino foram mais propensos a usar } \\
\text { álcool no último mês quando o primeiro uso de álcool ocorreu } \\
\text { antes dos } 13 \text { anos. }\end{array}$ \\
\hline $\begin{array}{l}\text { Jackson } \\
\text { et al. } \\
(2016)^{61}\end{array}$ & EUA & Longitudinal & $\begin{array}{c}931 \\
(10 \text { a } 15)\end{array}$ & $\begin{array}{c}\text { Consumo entre as meninas era } 49 \% \text { mais elevado que nos } \\
\text { meninos. Estresse percebido, histórico familiar de problemas } \\
\text { com bebida e o consumo atual dos pais, o divórcio/separação } \\
\text { aumentou significativamente as chances de iniciação. }\end{array}$ \\
\hline $\begin{array}{l}\text { Adrian et al. } \\
(2016)^{62}\end{array}$ & AUSTRÁLIA & $\begin{array}{l}\text { Inquérito } \\
\text { domiciliar }\end{array}$ & $\begin{array}{c}7.357 \\
(14 \text { e } 17)\end{array}$ & $\begin{array}{l}\text { Adolescentes relataram que amigos e pais foram as fontes de } \\
\text { sua primeira bebida alcoólica, com taxas de prevalência muito } \\
\text { semelhantes de } 1998 \text { a } 2007 \text { (variação de } 24,3 \% \text { a } 27,9 \% \text { ). Irmãos } \\
\text { e outras fontes eram relativamente raros como fonte de primeira } \\
\text { bebida alcoólica. Em } 2010 \text { e } 2013 \text {, houve uma queda significativa e } \\
\text { significativa }(p<0,05 \text { ) na proporção de adolescentes que relataram } \\
\text { que os pais forneceram sua primeira bebida alcoólica - de } 24,3 \% \\
\text { em } 2007 \text { para } 15,0 \% \text { e } 13,9 \% \text { em } 2010 \text { e } 2013 \text {, respectivamente. }\end{array}$ \\
\hline $\begin{array}{l}\text { Li et al. } \\
(2014)^{63}\end{array}$ & $\begin{array}{l}\text { NOVA } \\
\text { ZELÂNDIA }\end{array}$ & Longitudinal & $\begin{array}{c}2.179 \\
(16 \text { e } 17)\end{array}$ & $\begin{array}{c}\text { Monitoramento particularmente pelos pais, era protetor contra o } \\
\text { uso de álcool. }\end{array}$ \\
\hline $\begin{array}{l}\text { Murphy } \\
\text { et al. } \\
(2016)^{64}\end{array}$ & IRLANDA & Transversal & $\begin{array}{c}982 \\
\text { (Mediana } 17 \\
\text { anos) }\end{array}$ & 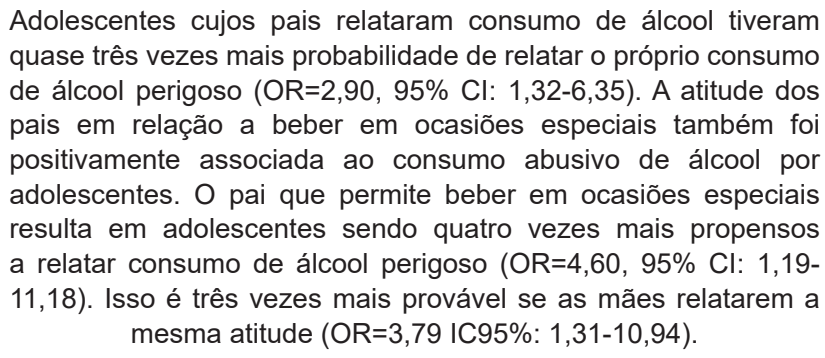 \\
\hline $\begin{array}{l}\text { Puente } \\
\text { et al. } \\
(2016)^{65}\end{array}$ & MÉXICO & Descritivo & $\begin{array}{c}386 \\
(15 \text { a } 20)\end{array}$ & $\begin{array}{l}\text { Idade média de início de consumo de álcool de } 14,1 \text { anos; a } \\
\text { maioria é do sexo masculino do que do sexo feminino ( } p= \\
0,009) \text {. Diferenças significativas entre mulheres e homens na taxa } \\
\text { de frequência e quantidade ( } p=0,003 \text { ) e na taxa de consumo } \\
\text { dependente }(p=0,006) \text {, com a maioria dos homens do que das } \\
\text { mulheres. Em relação à quantidade de bebida alcoólica consumida } \\
\text { em um dia típico, os homens consumiram mais que as mulheres, } \\
\text { mostrando uma diferença significativa }(p=0,006) \text {. }\end{array}$ \\
\hline $\begin{array}{l}\text { Vieira et al. } \\
(2008)^{66}\end{array}$ & BRASIL & Transversal & $\begin{array}{c}1.170 \\
(13 \text { a } 15)\end{array}$ & $\begin{array}{l}\text { Alto índice de uso pelos pais dos adolescentes, } 71 \% \text { consumiam } \\
\text { bebidas alcoolicas, prevalência maior do consumo dos } \\
\text { adolescentes aconteceu junto da família }(30,7 \%) \text { e dos amigos } \\
(34,5 \%) \text {; associação entre o uso de álcool pelos pais e o uso pelo } \\
\text { menos uma vez na vida pelos escolares foi significativa }(p<0,000) \text {. } \\
\text { Em relação às variáveis sexo, cor da pele, classificação sócio- } \\
\text { econômica e percepção da imagem corporal, não se encontrou } \\
\text { associação com os desfechos investigados. }\end{array}$ \\
\hline
\end{tabular}


*continuação.

\begin{tabular}{|c|c|c|c|c|}
\hline $\begin{array}{l}\text { Monika } \\
\text { et al. } \\
(2016)^{67}\end{array}$ & AUSTRÁLIA & Longitudinal & $\begin{array}{c}1729 \\
(12,9 \pm 0,5)\end{array}$ & $\begin{array}{l}\text { Oferta de álcool aconteceu em contextos familiares, feriados, } \\
\text { jantares familiares e festas; os adolescentes tinham acesso } \\
\text { domiciliar ao álcool }(O R=1,08) \text {, uso dos pais }(O R=1,02) \text {. }\end{array}$ \\
\hline $\begin{array}{l}\text { Atilola et al. } \\
(2013)^{68}\end{array}$ & NÍGÉRIA & Transversal & $\begin{array}{c}587 \\
(15.1 \pm 1.4)\end{array}$ & $\begin{array}{l}\text { Fatores que foram independentemente associados ao uso de } \\
\text { álcool e outras substâncias no presente estudo foram fatores } \\
\text { pessoais como idade avançada, sexo masculino e baixo } \\
\text { desempenho acadêmico. O único fator relacionado à família que } \\
\text { foi independentemente associado ao uso de álcool e substâncias } \\
\text { por adolescentes no presente estudo foi o uso parental de álcool. }\end{array}$ \\
\hline $\begin{array}{l}\text { Bekman } \\
\text { et al. } \\
(2010)^{69}\end{array}$ & CALIFÓRNIA & Transversal & $\begin{array}{c}1.298 \\
(14 \text { a 18) }\end{array}$ & $\begin{array}{l}\text { Os estudantes do sexo masculino tiveram percepções ligeiramente } \\
\text { maiores de quantas bebidas médias seus pares teriam por ocasião } \\
\text { de consumo do que as alunas do sexo feminino; outras variáveis } \\
\text { não respondem à pergunta. }\end{array}$ \\
\hline $\begin{array}{l}\text { Bellis et al. } \\
(2009)^{70}\end{array}$ & INGLATERRA & Ecológico & $\begin{array}{c}9.833 \\
(15 \text { e } 16)\end{array}$ & $\begin{array}{c}\text { Acesso ao álcool pelos pais, ter renda semanal, beber em locais } \\
\text { fora de casa foram os fatores associados ao consumo do álcool e } \\
\text { aos resultados adversos apresentados por ele. }\end{array}$ \\
\hline $\begin{array}{l}\text { Castro et al. } \\
(2009)^{71}\end{array}$ & EUA & Transversal & $\begin{array}{c}945 \\
(10 \text { a 17) }\end{array}$ & $\begin{array}{l}\text { Uso de álcool altamente correlacionado para os meninos }(p<0,8) \\
\text { e para meninas }(p<0,69) \text {. Orgulho étnico e aculturação foram } \\
\text { associados com menor uso de substâncias entre meninos e } \\
\text { meninas. }\end{array}$ \\
\hline $\begin{array}{l}\text { Chen e } \\
\text { Jacobson } \\
(2012)^{72}\end{array}$ & EUA & Longitudinal & $\begin{array}{c}15.701 \\
(12 \text { a } 33)\end{array}$ & $\begin{array}{l}\text { Caucasianos apresentaram taxas mais altas de mudança e } \\
\text { tiveram os maiores níveis de uso de substâncias desde a metade } \\
\text { da adolescência. }\end{array}$ \\
\hline $\begin{array}{l}\text { Chun et al. } \\
(2008)^{73}\end{array}$ & EUA & Ensaio clínico & $\begin{array}{c}160 \\
(13 \text { a 17) }\end{array}$ & $\begin{array}{l}\text { Níveis significativamente mais altos de uso de substâncias } \\
\text { em homens da adolescência média até a idade adulta jovem. } \\
\text { Aos } 12 \text { anos, os adolescentes hispânicos exibiram níveis } \\
\text { significativamente mais altos de todas as formas de uso de } \\
\text { substâncias do que outros grupos raciais/étnicos; os adolescentes } \\
\text { asiáticos relataram níveis iniciais significativamente mais baixos } \\
\text { de uso de álcool e bebida alcoólica; indivíduos afro-americanos } \\
\text { geralmente apresentam as menores taxas de mudança no uso de } \\
\text { álcool, consumo excessivo de álcool. }\end{array}$ \\
\hline $\begin{array}{l}\text { Dickens } \\
\text { et al. } \\
(2012)^{74}\end{array}$ & EUA & Transversal & $\begin{array}{c}2.582 \\
(12 \text { a 19) }\end{array}$ & $\begin{array}{l}\text { O uso de pares está positivamente associado ao nível de uso de } \\
\text { álcool, e o vínculo escolar está negativamente associado ao nível } \\
\text { de uso de álcool. }\end{array}$ \\
\hline $\begin{array}{l}\text { Bachman } \\
\text { et al. } \\
(2011)^{75}\end{array}$ & MICHIGAN & $\begin{array}{l}\text { Inquérito } \\
\text { escolar }\end{array}$ & $\begin{array}{l}360.000 \\
(13 \text { a } 18)\end{array}$ & $\begin{array}{l}\text { Casos de consumo excessivo (cinco ou mais bebidas seguidas } \\
\text { pelo menos uma vez nas últimas duas semanas) são muito } \\
\text { menos prováveis entre os alunos do } 8^{\circ} \text { ano com pais altamente } \\
\text { qualificados, comparados com aqueles com menos pais educados. } \\
\text { na } 12^{\mathrm{a}} \text { série, na verdade, é levemente revertida, com casos de } \\
\text { consumo pesado um pouco menos prováveis entre aqueles com } \\
\text { pais menos instruídos. Entre os alunos da } 12^{\mathrm{a}} \text { série hispânicos, } \\
\text { há até mesmo alguma sugestão de uma relação positiva entre a } \\
\text { educação dos pais e os casos de consumo excessivo de álcool. }\end{array}$ \\
\hline $\begin{array}{l}\text { Banks e } \\
\text { Zapolski } \\
(2016)^{76}\end{array}$ & EUA & Transversal & $\begin{array}{c}104 \\
(12 \text { a 18) }\end{array}$ & $\begin{array}{l}\text { Análises preliminares revelaram diferenças no uso de álcool } \\
\text { por idade }(p=0,023) \text {, mas não sexo ou local de recrutamento. } \\
\text { As expectativas de álcool não diferiram por raça ou qualquer } \\
\text { covariável. revelaram que a raça moderou significativamente a } \\
\text { relação entre as expectativas do álcool na adolescência }\end{array}$ \\
\hline
\end{tabular}


*continuação.

\begin{tabular}{|c|c|c|c|c|}
\hline $\begin{array}{l}\text { Birhanu } \\
\text { et al. } \\
(2014)^{77}\end{array}$ & WOREATA & Transversal & $\begin{array}{c}651 \\
(14 \text { a 18) }\end{array}$ & $\begin{array}{l}\text { Uso de substâncias por irmãos (AOR [IC 95\%]: } 2,72[1,79,4,14] \text { ), } \\
\text { história familiar de uso de álcool e substâncias (AOR [IC 95\%] 2,24 } \\
[1,39-3,59]) \text { e uso de substâncias pelos amigos (AOR [IC 95\%] 2,14 } \\
{[1,44-3,18] \text { ) foram fatores positivamente associados ao uso de }} \\
\text { substâncias. Por outro lado, a religiosidade e a habilidade social, } \\
\text { foram encontradas em } 54 \% \text { (AOR [95\% CI] 0,46, }[0,31-0,68] \text { ) } \\
39 \% \text { (AOR [95\% CI] } 0,6[0,40-0,91] \text { ) associado negativamente } \\
\text { com uso de substâncias. }\end{array}$ \\
\hline $\begin{array}{l}\text { Brooks- } \\
\text { Russell } \\
\text { et al. } \\
(2015)^{78}\end{array}$ & EUA & Longitudinal & $\begin{array}{c}2.512 \\
(15 \text { a } 18)\end{array}$ & $\begin{array}{l}\text { O conhecimento de monitoramento parental e a desaprovação } \\
\text { parental percebida eram protetores para o uso de substâncias } \\
\text { O conhecimento de monitoramento dos pais foi associado com } \\
\text { menor probabilidade de associação. }\end{array}$ \\
\hline $\begin{array}{l}\text { Cheadle e } \\
\text { Whitbeck } \\
(2012)^{79}\end{array}$ & $\begin{array}{l}\text { ESTADOS } \\
\text { UNIDOS E DO } \\
\text { CANADÁ }\end{array}$ & Longitudinal & $\begin{array}{c}727 \\
(10 \mathrm{a} \mathrm{17})\end{array}$ & $\begin{array}{l}\text { Aumento do uso de bebidas alcoólicas aos } 11-12 \text { anos de } \\
\text { idade; meninas bebiam mais cedo que os meninos pelo RR de } \\
60 \% \text {; estresse financeiro, abuso de álcool por parte dos pais e } \\
\text { depressão maior dos pais predizem a ingestão precoce; eventos } \\
\text { de vida negativos e discriminação percebida pelos adolescentes } \\
\text { também estão associados. Amizades aumenta o risco de início } \\
\text { precoce do consumo de bebidas alcoólicas por um fator de } 2,6 \text { e } \\
\text { aumenta o risco de início na adolescência em } 50 \% \text {. Percepções } \\
\text { da criança sobre a aprovação dos pais são protecionistas. }\end{array}$ \\
\hline $\begin{array}{l}\text { Clark et al. } \\
(2016)^{80}\end{array}$ & CALIFÓRNIA & Longitudinal & $\begin{array}{c}674 \\
(14,27 \pm \\
0,53)\end{array}$ & $\begin{array}{l}\text { O monitoramento dos pais (especialmente quando percebido pela } \\
\text { criança) está associado ao uso de substâncias. O monitoramento } \\
\text { dos pais foi um preditor significativo dessas variáveis quando os } \\
\text { adolescentes estavam com pouco controle de esforço. }\end{array}$ \\
\hline $\begin{array}{l}\text { Cleveland } \\
\text { et al. } \\
(2011)^{81}\end{array}$ & $\begin{array}{c}\text { PENNSYLVA- } \\
\text { NIA }\end{array}$ & Longitudinal & $\begin{array}{c}48.641 \\
(11 \text { a } 18)\end{array}$ & $\begin{array}{l}\text { Maiores níveis de proteção familiar corresponderam à diminuição } \\
\text { da chance de uso (todos os ORs }<1,0, p<0,01 \text { ) o nível familiar } \\
\text { quanto a proteção agregada no nível da escola esteve } \\
\text { significativamente associada à diminuição das chances de uso de } \\
\text { substâncias (todos os } p<0,05 \text { ). }\end{array}$ \\
\hline $\begin{array}{l}\text { Cranford } \\
\text { et al. } \\
(2011)^{82}\end{array}$ & EUA & Longitudinal & $\begin{array}{l}148 \\
(9 \text { a } 11 \\
12 \text { a } 14 \\
15 \text { a } 17)\end{array}$ & $\begin{array}{l}\text { O consumo materno durante a infância média dos filhos previu } \\
\text { o número de dias de bebedeira na adolescência média; e o } \\
\text { alcoolismo paterno durante a infância média de seus filhos e as } \\
\text { expectativas de álcool dos adolescentes no início da adolescência } \\
\text { prediziam a frequência de intoxicação na adolescência média. }\end{array}$ \\
\hline $\begin{array}{l}\text { Dida et al. } \\
(2014)^{83}\end{array}$ & ETIOPIA & Transversal & $\begin{array}{c}603 \\
(15 \text { a 29) }\end{array}$ & $\begin{array}{l}\text { Sexo, idade e status de uso de substâncias do pai, da mãe, dos } \\
\text { irmãos e do melhor amigo dos entrevistados tiveram associação } \\
\text { com o uso de substâncias. Os entrevistados do sexo masculino } \\
\text { foram cerca de dez vezes mais em risco de praticar o uso de } \\
\text { substâncias em comparação com os entrevistados do sexo } \\
\text { feminino [odds ratio (AOR) ajustado } 11,37 \text {, intervalo de confiança } \\
\text { de } 95 \% \text { (IC) } 4,42-29,23 \text {. }\end{array}$ \\
\hline $\begin{array}{l}\text { Dos Reis e } \\
\text { Oliveira } \\
(2015)^{84}\end{array}$ & BRASIL & Transversal & $\begin{array}{c}638 \\
(13 \text { a 17) }\end{array}$ & $\begin{array}{l}\text { O consumo de risco associou-se à faixa etária (16 a } 17 \text { anos), } \\
\text { idade do primeiro consumo ( } \leq 12 \text { anos), maior renda familiar, } \\
\text { ambiente familiar conflituoso, não praticar uma religião e consumo } \\
\text { alcoólico dos pais. observou-se associação com a idade do } \\
\text { primeiro consumo ( } \leq 12 \text { anos), sendo odds ratio (OR=2,5 e } \\
\text { intervalo de confiança de } 95 \% \text {; IC95\%) } 1,4-4,4 \text {. }\end{array}$ \\
\hline $\begin{array}{l}\text { Duncan } \\
\text { et al. } \\
(2011)^{85}\end{array}$ & EUA & Longitudinal & $\begin{array}{c}256 \\
(13 \text { a } 20)\end{array}$ & $\begin{array}{l}\text { Risco uso de álcool pelos amigos e uso de pares normativo foram } \\
\text { associados positivamente com maiores taxas iniciais de uso de } \\
\text { álcool. } \\
\text { O uso inicial de álcool pelos pais e a mudança positiva no uso de } \\
\text { álcool pelos pais e amigos ao longo do tempo foram relacionados } \\
\text { a um aumento no uso de álcool dos } 13 \text { aos } 20 \text { anos de idade. }\end{array}$ \\
\hline
\end{tabular}




\begin{tabular}{|c|c|c|c|c|}
\hline $\begin{array}{l}\text { Duncan } \\
\text { et al. } \\
(2012)^{86}\end{array}$ & EUA & Longitudinal & $\begin{array}{c}405 \\
11 \text { e } 13\end{array}$ & $\begin{array}{l}\text { O uso de álcool aumentou com a idade, assim como o consumo } \\
\text { excessivo de álcool, a embriaguez, o uso de álcool pelos pares e } \\
\text { a facilidade de obter álcool. Menos afro-americanos comparados } \\
\text { aos brancos relataram consumo de álcool, consumo excessivo } \\
\text { de álcool, embriaguez, uso de álcool entre colegas e incentivo ao } \\
\text { álcool. }\end{array}$ \\
\hline $\begin{array}{l}\text { Galduróz et } \\
\text { al. }(2010)^{87}\end{array}$ & BRASIL & Transversal & $\begin{array}{c}48.155 \\
(10 \text { a } 18)\end{array}$ & $\begin{array}{l}\text { De todos os estudantes, } 4.286(8,9 \%) \text { relataram uso pesado de } \\
\text { álcool no mês anterior à entrevista. A análise de regressão logística } \\
\text { mostrou associação entre relacionamento regular ou ruim com o } \\
\text { pai }(\mathrm{OR}=1,46) \text { e a mãe }(\mathrm{OR}=1,61) \text { e uso pesado de álcool. } \\
\text { Seguir uma religião (OR }=0,83) \text { foi inversamente associado ao } \\
\text { consumo pesado de álcool. A prática esportiva e a mãe percebida } \\
\text { como uma pessoa "liberal" não tiveram significado no modelo. } \\
\text { No entanto, uma maior prevalência de uso pesado de álcool foi } \\
\text { observada entre os estudantes que trabalham. }\end{array}$ \\
\hline $\begin{array}{l}\text { Hemovich } \\
(2012)^{88}\end{array}$ & EUA & Longitudinal & $\begin{array}{c}4.173 \\
(12 \text { a } 18)\end{array}$ & $\begin{array}{c}\text { A renda familiar e o gênero da criança }(p<0,001) \text {, juntamente com } \\
\text { a estrutura familiar }(p<0.05) \text {, monitoramento parental afetado, } \\
\text { predisseram a percepção social e interpessoal dos adolescentes } \\
\text { sobre o uso de drogas }(p<0,001) \text {, e ambas as variáveis } \\
\text { anteciparam o consumo real de drogas dos adolescentes um ano } \\
\text { depois }(p<0,001) .\end{array}$ \\
\hline $\begin{array}{l}\text { Johnson } \\
\text { et al. } \\
(2009)^{89}\end{array}$ & EUA & Transversal & $\begin{array}{c}4.750 \\
\text { (Média 15,4) }\end{array}$ & $\begin{array}{l}\text { Alunos que eram brancos, tinham desempenho ruim na escola, } \\
\text { não esperavam concluir o ensino médio e tinham mais dinheiro } \\
\text { discricionário para gastar; amigos que se embriagavam } \\
\text { semanalmente; diferenças significativas nas atitudes em relação a } \\
\text { beber e fumar foram observadas entre co-usuários e não usuários. }\end{array}$ \\
\hline $\begin{array}{l}\text { Keyes et al. } \\
(2015)^{90}\end{array}$ & EUA & Longitudinal & $\begin{array}{c}14.101 \\
(12 \text { a } 21)\end{array}$ & $\begin{array}{l}\text { Pico de consumo aconteceu aos } 16 \text { anos; entre negros, o consumo } \\
\text { diminuiu no período de estudo; os brancos eram significativamente } \\
\text { mais propensos do que negros a usar álcool, engajar-se em beber } \\
\text { em risco e tinham mais bebidas por sessão, tanto masculino como } \\
\text { feminino; brancos tiveram maior frequência de embriaguez; }\end{array}$ \\
\hline $\begin{array}{l}\text { Lopez et al. } \\
(2009)^{91}\end{array}$ & ESPANHA & Longitudinal & $\begin{array}{c}361 \\
(13,5)\end{array}$ & $\begin{array}{l}\text { Apenas o uso da substância percebida pelos pares estava } \\
\text { diretamente relacionado ao uso de substâncias pelos próprios } \\
\text { adolescentes. Uma interação significativa foi encontrada entre } \\
\text { o monitoramento dos pais e o uso de pares vis-à-vis o uso de } \\
\text { substâncias, o que sugere que a relação entre o monitoramento } \\
\text { dos pais e o uso dos próprios adolescentes foi significativamente } \\
\text { mais forte entre os jovens que relataram que mais amigos usavam } \\
\text { substâncias. }\end{array}$ \\
\hline $\begin{array}{l}\text { Lopez- } \\
\text { Vergara } \\
\text { et al. } \\
(2017)^{92}\end{array}$ & EUA & Longitudinal & $\begin{array}{c}944 \\
(12 \text { e } 16)\end{array}$ & $\begin{array}{l}\text { Probabilidade de iniciação foram previstos pelo sexo feminino, } \\
\text { idade avançada e mais conflito parental, prevalência percebida } \\
\text { de consumo de pares e maiores níveis de procura de sensações. }\end{array}$ \\
\hline $\begin{array}{l}\text { Shijun et al. } \\
(2015)^{93}\end{array}$ & CHINA & Transversal & $\begin{array}{l}13.811 \\
(15,3)\end{array}$ & $\begin{array}{l}\text { Para os estudantes que não moravam com as mães, assim } \\
\text { como os estudantes em nível socioeconômico mais elevado, } \\
\text { as chances ajustadas de beber no passado e no presente eram } \\
\text { significativamente mais altas, comparadas àquelas que viviam } \\
\text { com os pais e o baixo nível socioeconômico. }\end{array}$ \\
\hline $\begin{array}{l}\text { Malone } \\
\text { et al. } \\
(2012)^{94}\end{array}$ & EUA & Longitudinal & $\begin{array}{c}8.984 \\
(12 \text { a } 19)\end{array}$ & $\begin{array}{l}\text { Adolescentes negros iniciam o uso de álcool em idades mais } \\
\text { avançadas do que os jovens brancos. Além disso, após o início, } \\
\text { os adolescentes brancos foram substancialmente mais propensos } \\
\text { do que os adolescentes negros a continuar relatando o uso de } \\
\text { álcool nos anos subseqüentes. }\end{array}$ \\
\hline
\end{tabular}




\begin{tabular}{|c|c|c|c|c|}
\hline $\begin{array}{l}\text { Malta et al. } \\
(2012)^{95}\end{array}$ & BRASIL & Transversal & $\begin{array}{l}60.973 \\
(\leqq 14)\end{array}$ & $\begin{array}{l}\text { Demonstrado que o consumo é maior entre adolescentes mais } \\
\text { velhos e meninas. Os estudantes pardos apresentaram menos } \\
\text { chances de uso, assim como aqueles que frequentam as escolas } \\
\text { públicas. Maiores chances de consumo de álcool entre os } \\
\text { estudantes que: não moram com o pai; não tenha refeições com a } \\
\text { mãe ou responsável durante a semana; ter pais ou responsáveis } \\
\text { que não estejam cientes de suas atividades de tempo livre; e perder } \\
\text { aulas sem o consentimento dos pais. A supervisão da família } \\
\text { também é importante para a prevenção de tal comportamento. }\end{array}$ \\
\hline $\begin{array}{l}\text { Malta et al. } \\
(2012)^{96}\end{array}$ & BRASIL & Transversal & $\begin{array}{c}109.104 \\
(13 \text { a } 14)\end{array}$ & $\begin{array}{l}\text { Mais elevado entre as meninas, experimentação de drogas foi } \\
\text { mais elevada entre os meninos; características da saúde mental } \\
\text { como a solidão e insônia estiveram associadas positivamente ao } \\
\text { uso do tabaco, bebidas alcoólicas e drogas ilícitas. Não ter amigos } \\
\text { associou-se positivamente ao uso do tabaco e drogas ilícitas, e } \\
\text { negativamente ao uso do álcool. }\end{array}$ \\
\hline $\begin{array}{l}\text { Manson } \\
\text { et al. } \\
(2010)^{97}\end{array}$ & FILADÉLFIA & Transversal & $\begin{array}{c}301 \\
(13 \text { e } 20)\end{array}$ & $\begin{array}{l}\text { Meninos apresentavam significativamente mais risco. Os } \\
\text { resultados indicam que as meninas mais jovens com redes } \\
\text { sociais protetoras em seus locais de risco e favoritos são menos } \\
\text { propensas a usar substâncias em comparação com as meninas } \\
\text { com redes menos protetoras nesses locais (OR }=0,94, \text { IC }= \\
0,89-0,99, p<0,05) \text {. Em contraste, os machos mais jovens não } \\
\text { pareciam colher efeitos protetores de suas redes sociais. Tanto } \\
\text { as mulheres mais velhas }(\mathrm{OR}=0,96, \mathrm{Cl}=0,93-0,99, \mathrm{p}<0,005) \\
\text { e os homens mais velhos (OR }=0,86, \mathrm{Cl}=0,74-0,99, \mathrm{p}<0,05) \\
\text { reduziram a probabilidade de uso de substâncias se tivessem } \\
\text { redes de proteção em seus lugares de risco em comparação com } \\
\text { aqueles com redes não-protetoras nesses locais. }\end{array}$ \\
\hline $\begin{array}{l}\text { Wang et al. } \\
(2013)^{98}\end{array}$ & CALIFÓRNIA & Longitudinal & $\begin{array}{c}1.284 \\
(12 \text { a } 18)\end{array}$ & $\begin{array}{l}\text { Consumo entre pares; com relação às influências dos pais, } \\
\text { adolescentes que experimentaram mais bebida em casa tinham } \\
\text { maior probabilidade de aumentar sua própria frequência de } \\
\text { consumo. Adolescentes com níveis mais altos de monitoramento } \\
\text { dos pais se envolvem em menos comportamentos de beber ao } \\
\text { longo do tempo, mas esse efeito só é significativo na pequena } \\
\text { amostra escolar }(b=-.39, p<.05) \text {. }\end{array}$ \\
\hline $\begin{array}{l}\text { Weaver et } \\
\text { al. }(2011)^{99}\end{array}$ & KANSAS & Ensaio clínico & $\begin{array}{c}680 \\
(14 \text { a 18) }\end{array}$ & $\begin{array}{l}\text { Em relação aos adolescentes negros, os adolescentes brancos } \\
\text { relataram maior uso de álcool pelos pares durante o ensino médio } \\
\text { e eram muito mais propensos a consumir álcool durante o ensino } \\
\text { médio. Normas gerais de pares no crescimento do ensino médio } \\
\text { e sétimo grau em normas de uso de álcool entre amigos próximos } \\
\text { foram preditivas de uma maior propensão a consumir álcool no } \\
\text { nono ano entre os adolescentes brancos. }\end{array}$ \\
\hline $\begin{array}{l}\text { Gaete e } \\
\text { Araya } \\
(2017)^{100}\end{array}$ & CHILE & Transversal & $\begin{array}{c}45.273 \\
(12 \text { e } 21)\end{array}$ & $\begin{array}{l}\text { Disponibilidade de dinheiro de bolso, mais tempo gasto com } \\
\text { amigos, falta de monitoramento dos pais, mau vínculo escolar, } \\
\text { intimidação de terceiros e menor percepção de risco do uso de } \\
\text { substâncias e preditores exclusivos (por exemplo, realização } \\
\text { escolar em testes nacionais) foram identificados. }\end{array}$ \\
\hline $\begin{array}{l}\text { Jackson } \\
\text { et al. } \\
(2015)^{101}\end{array}$ & EUA & Longitudinal & $\begin{array}{c}1.023 \\
(11 \text { a } 14)\end{array}$ & $\begin{array}{l}\text { Maior desempenho escolar diminui a chance de beber álcool; } \\
\text { aumento para aqueles com comportamentos problemáticos, busca } \\
\text { de sensações ou impulsividade; irmãos consumidores aumentam } \\
\text { a chance para o consumo; Conflito com ambos os pais também } \\
\text { aumentou a chance do consumo. Apoio social paterno diminui; } \\
\text { quanto mais forte a reação negativa que a criança antecipou } \\
\text { dos pais, maior a probabilidade de ter início do ato de beber. As } \\
\text { chances de beber eram maiores entre os jovens que relataram que } \\
\text { seus pais reagiriam fortemente ao consumo de álcool pelo filho. } \\
\text { Além disso, maior controle parental foi associado exclusivamente } \\
\text { com a redução da probabilidade de início do consumo. }\end{array}$ \\
\hline
\end{tabular}


*continuação.

\begin{tabular}{|c|c|c|c|c|}
\hline $\begin{array}{l}\text { Jackson } \\
\text { et al. } \\
(2016)^{102}\end{array}$ & EUA & Longitudinal & $\begin{array}{c}164 \\
x\end{array}$ & $\begin{array}{c}\text { Adolescentes relatam ter recebido com maior frequência a } \\
\text { oferta do álcool por familiares, alguns consomem em casa sem } \\
\text { permissão; oferta por amigos ou namorados. }\end{array}$ \\
\hline $\begin{array}{l}\text { Myers } \\
(2014)^{103}\end{array}$ & CALIFÓRNIA & Transversal & $\begin{array}{c}714 \\
(16,3 \pm 1,3)\end{array}$ & $\begin{array}{l}\text { O risco de influências sociais semelhantes ao uso de substâncias } \\
\text { aumenta em } 44 \%(p=0,01) \text {. Idade }(b=0,34 ; p=0,001) \text { e ser do } \\
\text { sexo feminino versus masculino }(b=-54 ; p=0,04) \text { também foram } \\
\text { significativamente preditivos da influência social dos pares para o } \\
\text { uso de substâncias. }\end{array}$ \\
\hline $\begin{array}{l}\text { Clark et al. } \\
(2013)^{104}\end{array}$ & EUA & Longitudinal & $\begin{array}{c}20.743 \\
(11 \text { a } 21)\end{array}$ & $\begin{array}{l}\text { Uso de álcool aos } 13 / 16 \text { anos de idade; brancos beberam álcool } \\
\text { mais precocemente que as outras raças; relacionamento próximo } \\
\text { entre pais e filhos está significativamente associado à idade do } \\
\text { primeiro uso. }\end{array}$ \\
\hline $\begin{array}{l}\text { Ramsoomar } \\
\text { et al. } \\
(2013)^{105}\end{array}$ & $\begin{array}{l}\text { ÁFRICA DO } \\
\text { SUL }\end{array}$ & Longitudinal & $\begin{array}{c}3.273 \\
(13 \text { a } 18)\end{array}$ & $\begin{array}{l}\text { Nas análises multivariadas, o sexo, a escolaridade materna e o } \\
\text { nível socioeconômico previram o consumo de álcool na vida adulta } \\
\text { e no início da adolescência, enquanto o sexo, a escolaridade } \\
\text { materna, o estado civil e o status socioeconômico foram preditivos } \\
\text { do mesmo no final da adolescência. }\end{array}$ \\
\hline
\end{tabular}

Com relação à faixa-etária, estudos apontam as idades mais prevalentes para o consumo quando, de 09 a 12 anos $^{16,42,79}$; de 12 a $14 \operatorname{anos}^{21,30,46,60,73,79,85,104}$; de 15 a $16 \operatorname{anos}^{31,58,84,90,104}$; de 17 a $19 \operatorname{anos}^{59,84}$.

Os fatores de proteção para o consumo de álcool por adolescentes, foram: ter religião ${ }^{14,84,87}$; apoio familiar alto $^{20,101}$; estar em uma escola ${ }^{54,81}$; ser de escola pública ${ }^{95}$; alto desempenho escolar e vínculo com a escola ${ }^{40,53,74,101}$.

Diante dos resultados obtidos, observa-se que há uma quantidade significativa de estudos disponíveis na literatura investigando as possíveis associações e explicações dos fatores que contribuem para o uso de bebidas alcoólicas por adolescentes, porém não houve na presente pesquisa estudos que nomearam os fatores sociais/contextuais/ individuais como DSS, mesmo que eles tenham tratado sobre desigualdades sociais, renda, escolaridade, rede de apoio, grupo de pares, vizinhança e afins para refletir as condições de vida dessa população. O que dificulta a percepção quanto à distinção dos DSS e a uniformidade na definição.

Apesar dos resultados obtidos nesse estudo, existem certas limitações do ponto de vista metodológico, estudos de corte transversal (prevalente na revisão), inviabiliza a causalidade entre efeito e exposição/incidência, entretanto apontam os fatores associados relevantes para a produção de conhecimento sobre a temática. O rol de pesquisas nessa perspectiva encontrados no Brasil demonstra que é necessário ampliar os estudos, visto que neste país houve apenas 14 estudos ${ }^{14,25,28,30,34,38,48,50,51,66,84,87,95,97}$ encontrados, enquanto nos Estados Unidos da América (EUA) foram 48 estudos, conforme Miranda et al., ${ }^{106}$ países da América do Norte (Estados Unidos da América e Canadá) e da Europa, são países desenvolvidos e os efeitos dos DSS são mais devastadores sobre a saúde da população nos países em desenvolvimento.

Outro fator limitante do presente estudo, deve-se ao fato de considerar apenas textos de livre acesso, uma restrição que pode ter reduzido a possibilidade de inclusão de trabalhos relevantes. Por fim, a heterogeneidade das pesquisas levou a impossibilidade da realização de análises quantitativas e o cálculo de medida sumarizadas, o que se respalda pela característica inerente aos DSS.

A presente investigação revelou maior número de artigos nos anos de $2012^{21,25,37,39,51,72,74,79,86,88,94,95,96} ; 2013^{16,22,36,53,54,55,59,68,98,1}$ ${ }^{04,105} ; 2015^{24,27,33,35,38,41,52,57,58,60,76,78,84,90,93,101} ; 2014^{31,42,47,48,63,78,83,103}$ $2016^{17,28,43,49,60,61,62,64,65,67,80,102}$ e $2017^{14,40,44,92,100}$, o que evidencia um interesse crescente dos pesquisadores quanto à contribuição dos fatores sociais como risco para o processo saúde-doença, demonstrando que nas últimas décadas têmse visto maior empenho dos pesquisadores em produzir materiais que possibilitem a compreensão da maneira como a sociedade e suas diferentes formas de organização social influenciam a saúde e o estado de bem estar ${ }^{107}$. Isso pode estar relacionado também pelas políticas globais de saúde, propostas pelas Nações Unidas em 2000, com 
ênfase em ações sobre a redução de pobreza, investimento educacional, controle de epidemias e outros ${ }^{108}$.

A maioria dos estudos aponta para uma frequência maior quando o consumo é realizado pelo sexo masculino comparado ao sexo femininoUm estudo relata que o sexo masculino apresentou dez vezes mais chance de consumir bebida alcoólica comparado ao feminino ${ }^{83}$, outros dois estudos justificam que esse gênero tem facilidade na obtenção de álcool em lojas e também através de amigos $^{48,57}$. Já para o gênero feminino há algumas pesquisas que encontraram a associação e demonstram também que esse gênero consome mais em casa $^{48}$.

A diversidade entre as definições para medir as faixas etárias nos estudos analisados foram limitações importantes que impediram estabelecer uma evolução temporal para a frequência e comparação de uso de álcool, e o que se pode evidenciar é a idade mínima encontrada dentre todos os estudos, sendo entre os 09 e 12 anos de idade ${ }^{16,42,79}$.

Com relação à raça-cor, nota-se que na literatura estudada, a cor branca demonstra-se prevalente comparada à do jovem negro, e um estudo demonstra ainda que o negro que vive com renda salarial mais baixa aumenta seu consumo quando sobe de classe social ${ }^{22}$, o que nos leva a determinar que o nível socioeconômico mais alto além de predizer o consumo ${ }^{15,16,18,22-27,31,33,34,51,84,88,93,105}$ na maioria dos estudos aparece como fator para o maior consumo de álcool. Explica-se, pois, que adolescentes com mais recursos econômicos poderiam ter maior probabilidade de gastar com o consumo alcoólico ${ }^{102}$ diferentemente dos adolescentes com menos recursos financeiros, com menos poder de compra.

Com relação ao tipo da escola, particular ou pública, não há consenso na literatura estudada onde há maior risco, e especula-se que escolas menores poderiam motivar o envolvimento escolar, assim como incentivar a conectividade com a escola ${ }^{101,108,109}$, aumentar a participação escolar e engajamento de adolescentes ${ }^{101,110}$ e apego com a escola ${ }^{101,111}$ protegendo contra o consumo alcoólico. Esse fato não acontece nas escolas maiores que propiciam ambientes menos coesos, onde os alunos não conseguem manter o mesmo apego e participação escolar ${ }^{16}$. A entrada no ensino médio parece ser um evento crítico $^{112}$ de desenvolvimento associado ao aumento do risco social para um maior uso de álcool ${ }^{20,41,46,89,99}$, devido ao aumento do estresse acadêmico ${ }^{47,61,62}$, menor acompanhamento dos professores, adultos e maior liberdade pessoal ${ }^{113}$. É uma época em que os adolescentes sentem maior pressão social para estabelecer grupos de pares ${ }^{114}$.

$\mathrm{Na}$ literatura, o hábito do consumo pelos amigos/ pares demonstrou forte associação para o consumo na adolescência, sendo consensual entre todos os

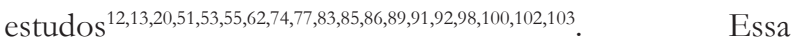
associação é um preditor estabelecido há muito tempo do uso de álcool pelos adolescentes, e os amigos parecem exercer grande influência para a progressão do uso, visto que os esses são mais suscetíveis à opinião e à avaliação dos seus pares ${ }^{115}$. Essa população por vezes busca a aprovação dos amigos, os quais podem transmitir mensagem de supervalorização do uso de álcool, o que contribui para o uso dessa substância.

O monitoramento dos pais demonstrou ser um fator de risco para o consumo caso esse seja norteado por uma supervisão deficiente entre todos os estudos, o que não acontece caso o adolescente tenha pais com alto índice de controle e de monitoramento ${ }^{56,63,78,91,98}$, levando a menor índice de consumo de álcool. Choukas-Bradley et al. ${ }^{19}$, indicaram que os pais que bebem são menos propensos a estabelecer regras contra a bebida para seus filhos, e isso também ocorre para pais que são favoráveis ao consumo de bebidas, indicando que as regras deles para o consumo de seus filhos têm uma influência mais forte do que seus comportamentos reais de bebida. Os pais que têm atitudes mais relaxadas em relação à bebida têm menos discussões relacionadas ao álcool do que os pais que têm atitudes rígidas em relação à bebida ${ }^{19}$. Quando a frequência de comunicação entre pais e filhos sobre o uso de bebidas alcoólicas é alta, os adolescentes são menos propensos a apresentar comportamentos problemáticos de bebida ${ }^{38}$.

Achados evidenciam que a experiência de divórcio dos pais foi associada ao início precoce do uso de álcool ${ }^{61}$, e esses achados em particular foram incluídos na revisão, pois tal acontecimento acomete diretamente os fatores psicológicos/comportamentais do adolescente. A literatura evidencia que o evento de separação dos pais resulta em um estresse percebido ${ }^{61}$, o que pode levar ao envolvimento com bebidas alcoólicas. Ainda, adolescentes de pais casados usam menos álcool que aqueles com famílias separadas ${ }^{61}$. Outra associação foi a violência, adolescentes expostos à violência, consomem bebidas alcoólicas mais precocemente que aqueles que não foram expostos ${ }^{48}$, e o uso explica-se provavelmente como mecanismo de enfrentamento ou que 
aqueles que estão em ambientes violentos tenham maior acesso às substâncias e sejam cercados por pares mais desviantes que reforçam comportamentos negativos ${ }^{117}$.

Após a análise dos artigos encontrados, nota-se que muito se tem avançado na análise entre a situação de saúde, a organização social dessa população, e, consequentemente, como essas relações influenciam para o consumo de álcool. São diversos os fatores atuantes para o uso de bebidas alcoólicas pelos adolescentes, e alguns deles são mais prevalentes que outros, o que permite o delineamento das causas que colaboram para esse consumo. Ainda, identificar, conhecer e entender tais influências colabora para a compreensão de como os DSS inserem-se na saúde dessa população.

\section{CONCLUSÃO|}

O presente estudo possibilitou a identificação dos Determinantes Sociais da Saúde associados ao consumo de álcool por adolescentes, sendo eles de ordens individuais, como gênero, idade, raça-cor, escolaridade, e de ordem contextual, como uso pelos pares, consumo feito pelos pais, escolaridade da mãe, presença materna e paterna quanto ao monitoramento dos filhos, assim como características ambientais. Isso evidencia a importância das pesquisas científicas na análise mais global e integrada da determinação social na produção e distribuição da doença. Essas análises são essenciais para subsidiar políticas e promoção da saúde. Os achados neste estudo demonstram a urgência da compreensão do cenário epidemiológico desse agravo a fim de reduzir essa taxa e prestar uma atenção qualificada aos indivíduos.

\section{REFERÊNCIAS |}

1. Covelo VMA. Adolescentes e o álcool numa escola do Alto Minho. Porto. Dissertação [Mestrado em Medicina] Universidade do Porto; 2013.

2. Lepre RM, Martins RA. Adolescente e a construção da identidade. Paidéia [Internet]. 2008; 19 [acesso em 12 mar 2012]. Disponível em: URL: <https://www.researchgate. net/publication/237343201_ADOLESCENCIA_E_ CONSTRUCAO_DA_IDENTIDADE $>$.
3. Alcohol, Drugs and Development Resources [Internet]. Harmful alcohol use is increasing in Americas: WHO Americas publishes alcohol and health report [acesso em 15 jan 2019]. Disponível em: URL: <http://www. add-resources.org/who-americas-publishes-alcohol-andhealth-report.5782868-315776.html>.

4. Puig-Nolasco A, Corteza-Ramirez L, Pillon SC. Consumo de alcohol entre estudiantes mexicanos de medicina. Rev Latino-Am Enfermagem. 2011; 19 (n. ${ }^{\circ}$ esp):714-21.

5. Trentini M, Paim L. Pesquisa convergente assistencial: um desenho que une o fazer e o pensar na prática assistencial em saúde-enfermagem. 2. ed. Florianópolis: Insular; 2004.

6. Brasil. Ministério da Saúde. Programa Saúde do Adolescente: bases programáticas. 2. ed. Brasília: Ministério da Saúde; 1996.

7. Brasil. Lei no. 8.069, de 13 de julho de 1990. Dispõe sobre o Estatuto da Criança e do Adolescente e dá outras providências [Internet]. Diário Oficial da União 16 jul 1990 [acesso em 07 mar 2009]. Disponível em: URL: <http:// www.planalto.gov.br/ccivil_03/LEIS/L8069.htm>.

8. Traverso-Yépez MA, Pinheiro VS. Adolescência, saúde e contexto social: esclarecendo práticas. Psicol Soc. 2002; 14(2):133-47.

9. Dahlgren G, Whitehead M. Policies and Strategies to promote social equity in health. Stockholm: Institute for Future Studies; 1991.

10. Silva VL, Cesse EAP, Albuquerque MFPM. Determinantes sociais da mortalidade do idoso: uma revisão sistemática da litteratura. Rev Bras Epidemiol. 2014; 17:178-93.

11. Moher D, Liberati A, Tetzlaff J, Altman DG; PRISMA Group. Preferred reporting items for systematic reviews and meta-analyses: the PRISMA statement. PLoS Med. 2009; 6(7):e1000097.

12. Chen MJ, Grube JW, Gruenewald PJ. Community alcohol outlet density and underage drinking. Addiction. 2010; 105(2):270-8.

13. Huckle T, Huakau J, Sweetsur P, Huisman O, Casswell S. Density of alcohol outlets and teenage drinking: living 
in an alcogenic environment is associated with higher consumption in a metropolitan setting. Addiction. 2008; 103(10):1614-21.

14. Jorge KO, Ferreira RC, Ferreira EF, Vale MP, Kawachi I, Zarza PM. Binge drinking and associated factors among adolescents in a city in southeastern Brazil: a longitudinal study. Cad Saúde Pública. 2017; 33(2):1-13.

15. Lu S, Du S, Hu X, Zou S, Liu W, Ba L, et al. Drinking patterns and the association between socio-demographic factors and adolescents' alcohol use in three metropolises in China. Int J Environ Res Public Health. 2015; 12(2):203753.

16. Andrade FH. Can the school context moderate the protective effect of parental support on adolescents' alcohol trajectories in urban Chicago? Drug Alcohol Depend. 2013; 133(2):330-7.

17. Azar D, White V, Coomber K, Faulkner A, Livingston M, Chikritzhs T, et al. The association between alcohol outlet density and alcohol use among urban and regional Australian adolescents. Addiction. 2016; 111(1):65-72.

18. Caria MP, Faggiano F, Bellocco R, Galanti MR. The influence of socioeconomic environment on the effectiveness of alcohol prevention among European students: a cluster randomized controlled trial. BMC Public Health. 2011; 11: 312.

19. Choukas-Bradley S, Giletta M, Neblett EW, Prinstein MJ. Ethnic differences in associations among popularity, likability, and trajectories of adolescents' alcohol use and frequency. Child Dev. 2015; 86(2):519-35.

20. Brenner AB, Bauermeister JA, Zimmerman MA. Neighborhood variation in adolescent alcohol use: examination of socioecological and social disorganization theories. J Stud Alcohol Drugs. 2011; 72(4):651-9.

21. Chen HJ, Balan S, Price RK. Association of contextual factors with drug use and binge drinking among white, native American, and mixed-race adolescents in the general population. J Youth Adolesc. 2012; 41(11):1426-41.

22. Clark TT, Corneille M, Coman E. Developmental trajectories of alcohol use among monoracial and biracial black adolescents and adults. J Psychoactive Drugs. 2013; 45(3):249-57.

23. Coomber K, Toumbourou JW, Miller P, Staiger PK, Hemphill SA, Catalano RF. Rural adolescent alcohol, tobacco, and illicit drug use: a comparison of students in Victoria, Australia, and Washington State, United States. J Rural Health. 2011; 27(4):409-15.

24. Hill D, Mrug S. School-level correlates of adolescent tobacco, alcohol, and marijuana use. Subst Use Misuse. 2015; 50(12):1518-28.

25. Locatelli D, Sanchez Z, Opaleye E, Carlini C, Noto A. Socioeconomic influences on alcohol use patterns among private school students in São Paulo. Rev Bras Psiquiatr. 2012; 34(2):193-200.

26. Lu S, Du S, Zhang Q, Hu X, Chen S, Wang Z, et al. Alcoholic beverage preferences and associated drinking patterns by socioeconomic status among high-school drinkers in three metropolises of China. Asia Pac J Clin Nutr. 2016; 25(1):184-94.

27. Lui CK, Chung PJ, Ford CL, Grella CE, Mulia N. Drinking behaviors and life course socioeconomic status during the transition from adolescence to adulthood among whites and blacks. J Stud Alcohol Drugs. 2015; 76(1):68-79.

28. Martins-Oliveira JG, Jorge KO, Ferreira RC, Ferreira EF, Vale MP, Zarzar PM. Risk of alcohol dependence: prevalence, related problems and socioeconomic factors. Ciênc Saúde Coletiva. 2016; 21(1):17-26.

29. Mrug S, Gaines J, Su W, Windle M. School-level substance use: effects on early adolescents' alcohol, tobacco, and marijuana use. J Stud Alcohol Drugs. 2010; 71(4):488-95.

30. Pinsky I, Sanches M, Zaleski M, Laranjeira R, Caetano R. Patterns of alcohol use among Brazilian adolescents. Rev Bras Psiquiatr. 2010; 32(3):242-9.

31. Poonawalla IB, Kendzor DE, Owen MT, Caughy MO. Family income trajectory during childhood is associated with adolescent cigarette smoking and alcohol use. Addict Behav. 2014; 39(10):1383-8. 
32. Primack BA, Kraemer KL, Fine MJ, Dalton MA. Media exposure and marijuana and alcohol use among adolescents. Subst Use Misuse. 2009; 44(5):722-39.

33. Fagan AA, Wright EM, Pinchevsky GM. Exposure to violence, substance use, and neighborhood context. Soc Sci Res. 2015; 49:314-26.

34. Ferreira LN, Sales ZN, Casotti CA, Bispo Júnior JP, Braga Júnior ACR. Alcohol consumption and associated factors in a city in Northeast Brazil. Cad Saúde Pública. 2011; 27(8):1473-86.

35. Sweeting H, Hunt K. Adolescent socioeconomic and school-based social status, smoking, and drinking. J Adolesc Health. 2015; 57(1):37-45.

36. Young R, Macdonald L, Ellaway A. Associations between proximity and density of local alcohol outlets and alcohol use among Scottish adolescents. Health Place. 2013; 19:124-30.

37. Brody GH, Kogan SM, Chen YF. Perceived discrimination and longitudinal increases in adolescent substance use: gender differences and mediational pathways. Am J Public Health. 2012; 102(5):1006-11.

38. Brito ALS, Hardmanb CM, Barros MVG. Prevalence and factors associated with the co-occurrence of health risk behaviors in adolescentes. Rev Paul Pediatr. 2015; 33(4):423-30.

39. Berten H, Cardoen D, Brondeel R, Vettenburg N. Alcohol and cannabis use among adolescents in Flemish secondary school in Brussels: effects of type of education. BMC Public Health. 2012; 12:215.

40. Bosque-Prous M, Kuipers MAG, Espelt A, Richter M, Rimpelä A, Perelman J, et al. Adolescent alcohol use and parental and adolescent socioeconomic position in six European cities. BMC Public Health. 2017; 17(1):646.

41. Andreas JB, Jackson KM. Adolescent alcohol use before and after the high school transition. Alcohol Clin Exp Res. 2015; 39(6):1034-41.

42. Chung T, Pedersen SL, Kim KH, Hipwell AE, Stepp SD. Racial differences in type of alcoholic beverage consumed during adolescence in the Pittsburgh Girls Study. Alcohol Clin Exp Res. 2014; 38(1):285-93.

43. D’Amico EJ, Tucker JS, Miles JN, Ewing BA, Shih RA, Pedersen ER. Alcohol and marijuana use trajectories in a diverse longitudinal sample of adolescents: examining use patterns from age 11 to 17 years. Addiction. 2016; 111(10):1825-35.

44. Davies LEM, Kuipers MAG, Junger M, Kunst AE. The role of self-control and cognitive functioning in educational inequalities in adolescent smoking and binge drinking. BMC Public Health. 2017; 17(1):714.

45. Donath C, Gräßel E, Baier D, Pfeiffer C, Karagülle $\mathrm{D}$, Bleich $\mathrm{S}$, et al. Alcohol consumption and binge drinking in adolescents: comparison of different migration backgrounds and rural vs. urban residence - a representative study. BMC Public Health. 2011; 11:84.

46. Ennett ST, Foshee VA, Bauman KE, Hussong A, Faris R, Hipp J, et al. The social ecology of adolescent alcohol misuse. Child Dev. 2008; 79(6):1777-91.

47. Liu X, Keyes K, Li G. Work stress and alcohol consumption among adolescents: moderation by family and peer influences. BMC Public Health. 2014; 14:1303.

48. Malta DC, Machado IE, Porto DL, Da Silva MMA, De Freitas PC, Da Costa AWN, et al. Alcohol consumption among Brazilian adolescents according to the National Adolescent School-based Health Survey (PeNSE 2012). Rev Bras Epidemiol. 2014; 17(Supl. 1):203-14.

49. Mejia R, Pérez A, Abad-Vivero EN, Kollath-Cattano C, Barrientos-Gutierrez I, Thrasher JF, et al. Exposure to alcohol use in motion pictures and teen drinking in Latin America. Alcohol Clin Exp Res. 2016; 40(3):631-7.

50. Noal RB, Menezes AMB, Araújo CL, Hallal PC. Experimental use of alcohol in early adolescence: the 11year follow-up of the 1993 Pelotas (Brazil) birth cohort study. Cad Saúde Pública. 2010; 26(10):1937-44.

51. Zarzar PM, Jorge KO, Oksanen T, Vale MP, Ferreira EF, Kawachi I. Association between binge drinking, type of friends and gender: a cross-sectional study among Brazilian adolescents. BMC Public Health. 2012; 12:257. 
52. Zenic N, Terzic A, Rodek J, Spasic M, Sekulic D. Genderspecific analyses of the prevalence and factors associated with substance use and misuse among Bosniak adolescents. Int J Environ Res Public Health. 2015; 12(6):6626-40.

53. Botticello AL. School contextual influences on the risk for adolescent alcohol misuse. Am J Community Psychol. 2009; 43(1-2):85-97.

54. Lo CC, Weber J, Cheng TC. Urban-rural differentials: a spatial analysis of Alabama students' recent alcohol use and marijuana use. Am J Addict. 2013; 22(3):188-96.

55. Chan GC, Kelly AB, Toumbourou JW, Hemphill SA, Young RM, Haynes MA, et al. Predicting steep escalations in alcohol use over the teenage years: agerelated variations in key social influences. Addiction. 2013; 108(11):1924-32.

56. Fulkerson JA, Pasch KE, Perry CL, Komro K. Relationships between alcohol-related informal social control, parental monitoring and adolescent problem behaviors among racially diverse urban youth. J Community Health. 2008; 33(6):425-33.

57. Ghandour L, Afifi R, Fares S, El Salibi N, Rady A. Time trends and policy gaps: the case of alcohol misuse among adolescents in Lebanon. Subst Use Misuse. 2015; 50(14):1826-39.

58. Gruenewald PJ, Treno AJ, Ponicki WR, Huckle T, Yeh LC, Casswell S. Impacts of New Zealand's lowered minimum purchase age on context-specific drinking and related risks. Addiction. 2015; 110(11):1757-66.

59. Haugland SH, Holmen TL, Ravndal E, Bratberg GH. Parental alcohol misuse and hazardous drinking among offspring in a general teenage population: gender-specific findings from the Young-HUNT 3 study. BMC Public Health. 2013; 13:1140.

60. Hung CC, Chang HY, Luh DL, Wu CC, Yen LL. Do parents play different roles in drinking behaviours of male and female adolescents? A longitudinal follow-up study. BMJ Open. 2015; 5:e007179.

61. Jackson KM, Rogers ML, Sartor CE. Parental divorce and initiation of alcohol use in early adolescence. Psychol Addict Behav. 2016; 30(4):450-61.
62. Kelly AB, Chan GC, Weier M, Quinn C, Gullo MJ, Connor JP, et al. Parental supply of alcohol to Australian minors: an analysis of six nationally representative surveys spanning 15 years. BMC Public Health. 2016; 16:325.

63. Li K, Simons-Morton BG, Brooks-Russell A, Ehsani J, Hingson R. Drinking and parenting practices as predictors of impaired driving behaviors among U.S. adolescents. J Stud Alcohol Drugs. 2014; 75(1):5-15.

64. Murphy E, O'Sullivan I, O'Donovan D, Hope A, Davoren MP. The association between parental attitudes and alcohol consumption and adolescent alcohol consumption in Southern Ireland: a cross-sectional study. BMC Public Health. 2016; 16:821.

65. Rodríguez Puente LA, Alonso Castillo BA, Alonso Castillo MM, Alonso Castillo MT, Armendàriz Garcìa NA, Oliva Rodrìguez NN. Consumption of alcohol and tobacco in adolescentes. Rev Eletrônica Saúde Mental Álcool Drog. 2016; 12(4):200-6.

66. Vieira PC, Aerts DRGC, Freddo SL, Bittencourt A, Monteiro L. Uso de álcool, tabaco e outras drogas por adolescentes escolares em município do Sul do Brasil. Cad Saúde Pública. 2008; 24(11):2487-98.

67. Wadolowski M, Hutchinson D, Bruno R, Aiken A, Najman JM, Kypri K, et al. Parents who supply sips of alcohol in early adolescence: a prospective study of risk factors. Pediatrics. 2016; 137(3):e20152611.

68. Atilola O, Ayinde O, Adeitan O. Beyond prevalence and pattern: problematic extent of alcohol and substance use among adolescents in Ibadan South-west Nigeria. Afr Health Sci. 2013; 13(3):777-84.

69. Bekman NM, Cummins K, Brown SA. Affective and personality risk and cognitive mediators of initial adolescent alcohol use. J Stud Alcohol Drugs. 2010; 71(4):570-80.

70. Bellis MA, Phillips-Howard PA, Hughes K, Hughes S, Cook PA, Morleo M, et al. Teenage drinking, alcohol availability and pricing: a cross-sectional study of risk and protective factors for alcohol-related harms in school children. BMC Public Health. 2009; 9:380.

71. Castro FG, Stein JA, Bentler PM. Ethnic pride, traditional family values, and acculturation in early cigarette 
and alcohol use among Latino adolescents. J Prim Prev. 2009; 30(3-4):265.

72. Chen P, Jacobson KC. Developmental trajectories of substance use from early adolescence to young adulthood: gender and racial/ethnic differences. J Adolesc Health. 2012; 50(2):154-63.

73. Chun TH, Sindelar-Manning H, Eaton CA, Lewander WJ, Spirito A. Parental factors influence teen alcohol use after an emergency department visit. Pediatr Emerg Care. 2008; 24(10):668-72.

74. Dickens DD, Dieterich SA, Henry KL, Beauvais F. School bonding as a moderator of the effect of peer influences on alcohol use among American Indian adolescents. J Stud Alcohol Drugs. 2012; 73(4):597-603.

75. Bachman JG, O’Malley PM, Johnston LD, Schulenberg JE, Wallace JM. Racial/ethnic differences in the relationship between parental education and substance use among U.S. 8th-, 10th-, and 12th-grade students: findings from the Monitoring the Future project. J Stud Alcohol Drugs. 2011; 72(2):279-85.

76. Banksa DE, Zapolskia TCB. Racial differences in the link between alcohol expectancies and adolescent drinking. Addict Behav. 2017; 67:34-7.

77. Birhanu AM, Bisetegn TA, Woldeyohannes SM. High prevalence of substance use and associated factors among high school adolescents in Woreta Town, Northwest Ethiopia: multi-domain factor analysis. BMC Public Health. 2014; 14(1):1186.

78. Brooks-Russell A, Conway KP, Liu D, Xie Y, Vullo GC, Li K, et al. Dynamic patterns of adolescent substance use: results from a nationally representative sample of high school students. J Stud Alcohol Drugs. 2015; 76(6):962-70.

79. Cheadle JE, Whitbeck LB. Alcohol use trajectories and problem drinking over the course of adolescence: a study of north American indigenous youth and their caretakers. J Health Soc Behav. 2011; 52(2):228-45.

80. Clark DA, Donnellan MB, Robins RW, Conger RD. Early adolescent temperament, parental monitoring, and substance use in Mexican-origin adolescents. J Adolesc. 2015; 41:121-30.
81. Cleveland MJ, Feinberg ME, Greenberg MT. Protective families in high- and low-risk environments: implications for adolescent substance use. J Youth Adolesc. 2010; 39(2):114-26.

82. Cranford JA, Zucker RA, Jester JM, Puttler LI, Fitzgerald HE. Parental alcohol involvement and adolescent alcohol expectancies predict alcohol involvement in male adolescents. Psychol Addict Behav. 2010; 24(3):386-96.

83. Dida N, Kassa Y, Sirak T, Zerga E, Dessalegn T. Substance use and associated factors among preparatory school students in Bale Zone, Oromia Regional State, Southeast Ethiopia. Harm Reduc J. 2014; 11:21.

84. Reis TG, Oliveira LCM. Pattern of alcohol consumption and associated factors among adolescents students of public schools in an inner city in Brazil. Rev Bras Epidemiol. 2015; 18(1):13-24.

85. Duncan SC, Gau JM, Duncan TE, Strycker LA. Development and correlates of alcohol use from ages 1320. J Drug Educ. 2011; 41(3):235-52.

86. Duncan SC, Strycker LA, Duncan TE. Alcohol use of African Americans and whites from ages 9-20: descriptive results from a longitudinal study. J Ethn Subst Abuse. 2012; 11(3):214-25.

87. Galduróz JC, Sanchez Zv, Opaleye ES, Noto AR, Fonseca AM, Gomes PL, et al. Factors associated with heavy alcohol use among students in Brazilian capitals. Rev Saude Publica. 2010; 44(2):267-73.

88. Hemovich V, Lac A, Crano WD. Understanding earlyonset drug and alcohol outcomes among youth: the role of family structure, social factors, and interpersonal perceptions of use. Psychol Health Med. 2011; 16(3):24967.

89. Johnson CC, Webber LS, Myers L, Boris NW, Berenson GS. Co-use of alcohol and tobacco among ninth-graders in Louisiana. Prev Chronic Dis. 2009; 6(3):A85.

90. Keyes KM, Vo T, Wall MM, Caetano R, Suglia SF, Martins SS, et al. Racial/ethnic differences in use of alcohol, tobacco, and marijuana: is there a cross-over from adolescence to adulthood? Soc Sci Med. 2015; 124:132-41. 
91. Lopez B, Wang W, Schwartz SJ, Prado G, Huang $\mathrm{S}$, Brown CH, et al. School, family, and peer factors and their association with substance use in Hispanic adolescents. J Prim Prev. 2009; 30(6):622-41.

92. Lopez-Vergara HI, Merrill JE, Janssen T, Jackson KM. Social and individual-level predictors of alcohol use initiation and escalation: replicating and extending tests of differential effects. J Stud Alcohol Drugs. 2017; 78(3):4527.

93. Lu S, Du S, Hu X, Zou S, Liu W, Ba L, et al. Drinking patterns and the association between socio-demographic factors and adolescents' alcohol use in three metropolises in China. Int J Environ Res Public Health. 2015; 12(2):203753.

94. Malone PS, Northrup TF, Masyn KE, Lamis DA, Lamont AE. Initiation and persistence of alcohol use in United States Black, Hispanic, and White male and female youth. Addict Behav. 2012; 37(3):299-305.

95. Malta DC, Porto DL, Melo FCM, Monteiro RA, Sardinha LMV, Lessa BH. Family and the protection from use of tobacco, alcohol, and drugs in adolescents, National School. Rev Bras Epidemiol. 2011; 14(1 Supl.):166-77.

96. Malta DC, Oliveira-Campos M, Prado RR, Andrade SSC, Mello FCM, Dias AJR, et al. Psychoactive substance use, family context and mental health among Brazilian adolescents, National Adolescent School-based Health Survey (PeNSE 2012). Rev Bras Epidemiol. 2014; 17 (Supl. 1):46-61.

97. Mason MJ, Valente TW, Coatsworth JD, Mennis J, Lawrence F, Zelenak P. Place-based social network quality and correlates of substance use among urban adolescents. J Adolesc. 2010; 33(3):419-27.

98. Wang C, Hipp JR, Butts CT, Jose R, Lakon CM. Alcohol use among adolescent youth: the role of friendship networks and family factors in multiple school studies. PLoS One. 2015; 10(3):e0119965.

99. Weaver SR, Cheong J, MacKinnon DP, Pentz MA. Investigating ethnic differences in adolescent alcohol use and peer norms using semi-continuous latent growth models. Alcohol Alcohol. 2011; 46(5):620-6.
100. Gaete J, Araya R. Individual and contextual factors associated with tobacco, alcohol, and cannabis use among Chilean adolescents: a multilevel study. J Adolesc. 2017; 56:166-78.

101. Jackson KM, Colby SM, Barnett NP, Abar CC. Prevalence and correlates of sipping alcohol in a prospective middle school sample. Psychol Addict Behav. 2015; 29(3):766-78.

102. Jackson KM, Merrill JE, Barnett NP, Colby SM, Abar CC, Rogers ML, et al. Contextual influences on early drinking: characteristics of drinking and nondrinking days. Psychol Addict Behav. 2016; 30(5):566-77.

103. Myers R, Chou C, Sussman S, Baezconde-Garbanati L, Pachon H, Valente TW. Acculturation and substance use: social influence as a mediator among Hispanic alternative high school youth. J Health Soc Behav. 2009; 50(2):164-79.

104. Clark TT, Doyle O, Clincy A. Age of first cigarette, alcohol, and marijuana use among U.S. biracial/ethnic youth: a population-based study. Addict Behav. 2013; 38(9): 2450-4.

105. Ramsoomar L, Morojele NK, Norris AS. Alcohol use in early and late adolescence among the birth to twenty cohort in Soweto, South Africa. Glob Health Action. 2013; 6: 19274.

106. Miranda SS, Santos LPS, Araújo TM, Passos-Soares JS, Cruz SS, Gomes-Filho IS. A produção científica sobre determinantes sociais e condições de saúde: um estudo bibliométrico. Rev Ciênc Méd Biol. 2016; 15(2):208-13.

107. Krieger N. A glossary of social epidemiology. J Epidemiol Community Health. 2001; 55(10):693-700.

108. Organização Mundial de Saúde. Uso de álcool e comportamento sexual de risco: um estudo transcultural em oito países. Genebra: OMS; 2005.

109. Lee V, Smith JB. Efeitos da reestruturação do ensino médio e tamanho nos ganhos iniciais em conquistas e engajamento. Sociologia da Educação. 1995; 68:241-70.

110. McNeely CA, Nonnemaker JM, Blum RWJ. Promovendo a conectividade escolar: evidências do 
Estudo Nacional Longitudinal de Saúde do Adolescente. Sch Health. 2002; 72(4):138-46.

111. Silinsa H, Mulfordb B. Escolas como organizações de aprendizagem: efeitos na liderança do professor e nos resultados dos alunos. Eficácia escolar e melhoria escolar: Um Jornal Internacional de Pesquisa, Política e Prática. 2004; 15(3):443-66.

112. Crosnoe R, Johnson M, Elder GHJ. Tamanho da escola e o lado interpessoal da educação: um exame de raça/etnia e contexto organizacional. Ciências Sociais Trimestral. 2004; 85(5):1259-74.

113. Benner AD. A transição para o ensino médio: conhecimento atual, direções futuras. Rev Psicol Educ. 2011; 23:299-328.

114. Gillock KL, Reyes O. Mudanças no ensino médio relacionadas à transição no desempenho acadêmico de estudantes de minorias urbanas e percepções de si e do ambiente escolar. J Psicol Comun. 1996; 24:245-61.

115. Maxwell KA. Amigos: o papel da influência dos pares nos comportamentos de risco dos adolescentes. Jornal da Juventude e Adolescência. 2002; 31:267-77.

116. Duan L, Chou-CP, Andreeva VA, Ann-Pentz M. Trajetórias de influências sociais de pares como preditores a longo prazo do uso de drogas desde o início até o final da adolescência. Jornal da Juventude e Adolescência. 2009; 38:454-65.

117. Hong JS, Huang H, M Ouro, Upton Patton $\mathrm{D}$, Washington $\mathrm{T}$. Os jovens expostos à violência na comunidade correm o risco de se envolver em comportamento delinqüente? Uma revisão e implicações para pesquisa e prática de tratamento residencial. Tratamento Residencial para Crianças e Jovens. 2014; 31(4):266-83.
Correspondência para/Reprint request to:

\section{Fernanda Garcia Gabira}

Departamento de Enfermagem,

Centro de Ciências da Saúde,

Universidade Federal do Espirito Santo,

Avenida Marechal Campos, 1468,

Maruipe, Vitoria/ES, Brasil

CEP: 29060-270

Tel.: (27) 98133-4055

E-mail:fernandagabirag@gmail.com

Submetido em: 02/02/2019

Aceito em: 29/03/2019 